\title{
IMPLICIT AND EXPLICIT LEARNING OF A SERIAL REACTION TIME TASK
}

\section{CIENTRE FOR NEWIOOINIDLAND STUDIES}

TOTAL OF 10 PAGES ONLY MAY BE XEROXED

(Without Author's Permission)

RIOR M. HEALEY 



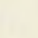


National Library

of Canada

Acquisitions and

Bibliographic Services

395 Wellington Street Ottawa ONK1A 0N4

Canada
Bibliothèque nationale

du Canada

Acquisisitons et services bibliographiques

395 , rue Wellington Ottawa ON K1A ON4

Canada
Yourfile Votre référence ISBN: 0-612-93032-7 Our file Notre référence ISBN: 0-612-93032-7
The author has granted a nonexclusive licence allowing the National Library of Canada to reproduce, loan, distribute or sell copies of this thesis in microform, paper or electronic formats.

The author retains ownership of the copyright in this thesis. Neither the thesis nor substantial extracts from it may be printed or otherwise reproduced without the author's permission.
L'auteur a accordé une licence non exclusive permettant à la Bibliothèque nationale du Canada de reproduire, prêter, distribuer ou vendre des copies de cette thèse sous la forme de microfiche/film, de reproduction sur papier ou sur format électronique.

L'auteur conserve la propriété du droit d'auteur qui protège cette thèse. $\mathrm{Ni}$ la thèse ni des extraits substantiels de celle-ci ne doivent être imprimés ou aturement reproduits sans son autorisation.
In compliance with the Canadian

Privacy Act some supporting forms may have been removed from this dissertation.

While these forms may be included in the document page count, their removal does not represent any loss of content from the dissertation.
Conformément à la loi canadienne sur la protection de la vie privée, quelques formulaires secondaires ont été enlevés de ce manuscrit.

Bien que ces formulaires aient inclus dans la pagination, il n'y aura aucun contenu manquant. 



\title{
IMPLICIT AND EXPLICIT LEARNING OF A SERIAL REACTION TIME TASK
}

by

\author{
Rick M. Healey
}

\author{
A thesis submitted to the \\ School of Graduate Studies \\ in partial fulfilment of the \\ requirements for the degree of \\ Masters of Science
}

Department of Psychology

Memorial University of Newfoundland

April, 2003

St. John's

Newfoundland 


\begin{abstract}
The performance of children in a serial reaction time task was studied. The independent variables were age (third and sixth graders), stimulus sequence ( 3 levels of structural complexity), stimulus type (asterisks and letters), and stimulus type sequence (letterasterisk and asterisk-letter). Explicit knowledge was assessed using verbal reports, recognition memory, and a generation task. Children's serial reaction times decreased as a function of verbal awareness and structure, but not as a function of age. Children's implicit sequence knowledge did not appear to transfer across different perceptual stimuli associated with the same response locations. There was some indication that participants learned complex four-trial part sequences implicitly. Participants presented with the high, as compared to the moderate, structure sequence were more likely to explicitly learn segments of the sequences, particularly series and bigrams/dyads. Measures of explicit learning appear to be related to a common explicit knowledge base. These findings are interpreted as reflecting implicit and explicit learning occur in parallel and that age invariance and transfer specificity of implicit knowledge may characterize middle childhood.
\end{abstract}




\section{Table of Contents}

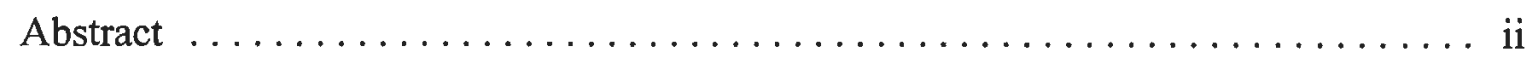

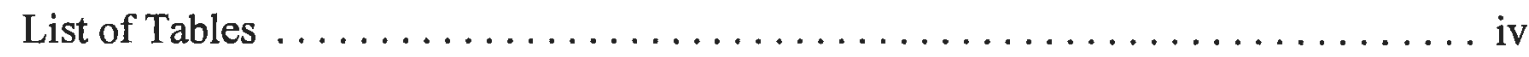

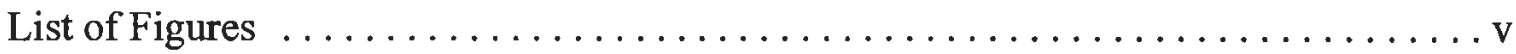

Acknowledgments $\ldots \ldots \ldots \ldots \ldots \ldots \ldots \ldots \ldots \ldots \ldots \ldots \ldots \ldots \ldots$ vi

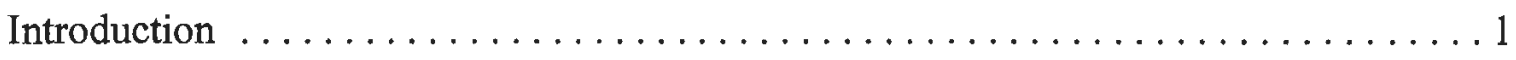

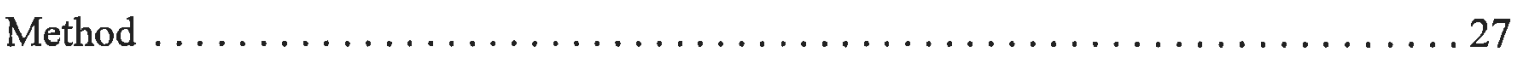

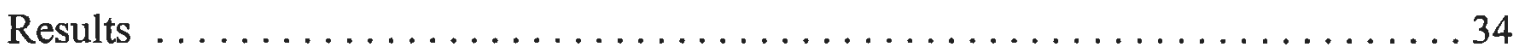

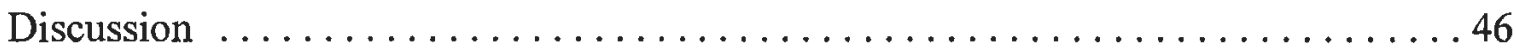

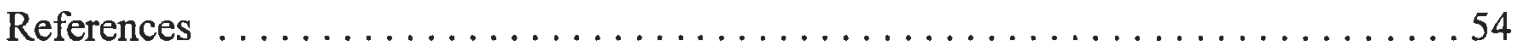

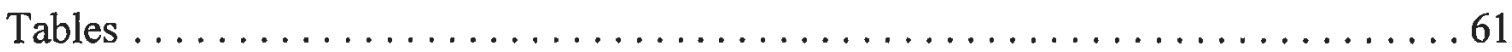

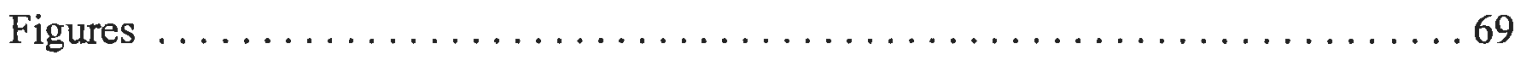


List of Tables

Table $1 \quad$ Summary of experimental design $\ldots \ldots \ldots \ldots \ldots \ldots \ldots \ldots \ldots \ldots \ldots$

Table 2 Number of children reporting they were aware, partially aware, or unaware of the sequential structure $\ldots \ldots \ldots \ldots \ldots \ldots \ldots \ldots \ldots \ldots \ldots \ldots \ldots$

Table 3 Mean serial reaction time (SD) in seconds for each sequential structure group as a function of stimulus-type sequence and the order in which each stimulus sequence was experienced $\ldots \ldots \ldots \ldots \ldots \ldots$

Table 4 Number of SRT errors across trial blocks as a function of stimulus type 64 Table 5 Mean judgement ratings of aware, partially aware, and unaware children with old, new, and ringer sequences $\ldots \ldots \ldots \ldots \ldots \ldots \ldots \ldots \ldots \ldots \ldots \ldots$

Table 6 Number of generation errors of aware, partially aware, and unaware children averaged across each 10 - trial block $\ldots \ldots \ldots 66 \ldots 6$

Table $7 \quad$ Correlations between explicit measures $\ldots \ldots \ldots \ldots \ldots \ldots$

Table 8 The frequency of participants who were errorless on the recognition task or on the first trial block of the generation task $\ldots \ldots \ldots \ldots$ 


\section{List of Figures}

Figure 1 The SRT task average median RT on each trial block as a function of age

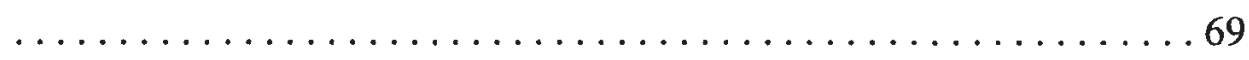

Figure 2 The SRT task average median RT on each trial block as a function of sequence structure 


\section{Acknowledgments}

I would like to thank my wife, Josie, and my children, Michael and Katelyn who provided me with more support than anyone could ever ask for throughout this project. I would also like to sincerely thank Dr. F. Michael Rabinowitz, my thesis supervisor, whose patience and guidance was immeasurable. 
Introduction

In the serial reaction time (SRT) task introduced by Nissen and Bullemer (1987), an asterisk appears on successive trials in one of four horizontal positions. Participants are required to press one of four buttons located below the asterisk as quickly and accurately as possible. The asterisk is displayed until the correct button is pressed. Following a correct button press, the asterisk is extinguished and a new asterisk appears in another position. Some participants experience asterisks that appear in a particular repeating order of positions while others experience asterisks that are presented in a quasi-random order such that asterisks appear unpredictably, but in a different position, on successive trails. Nissen and Bullemer reported participants who experienced a repeating sequence of asterisks decreased their reaction times (RTs) across trial blocks faster than participants who experienced a quasi-random sequence. This decrease in RT was considered an indirect measure of learning the repeating sequence. A number of researchers have claimed that most participants learn but do not become aware of the repeating sequence (Cherry \& Stadler, 1995; Cohen, Ivry, \& Keele, 1990; Curran, 1997; Frensch \& Miner, 1994; Hartman, Knopman, \& Nissen, 1989; Knopman \& Nissen, 1991; Mayr, 1996; Meulemans, Van der Linden, \& Perruchet, 1998; Nissen, Willingham, \& Hartman, 1989; Stadler, 1992; Stadler \& Neely, 1997; Willingham \& Dumas, 1997; Willingham, Greeley, \& Bardon, 1993; Willingham, Nissen, \& Bullemer, 1989). Although the researchers cited above contend learning can occur without awareness, some maintain that both implicit and explicit learning occur in parallel (Curran and Keele, 1993; Noseworthy, 1996; Seger, 1994; Sloman, 1996; Willingham, 2001; Willingham \& Goedert-Eschmann, 1999; Willingham, Salidis, \& Gabrieli, 2002), and others argue all learning is likely explicit (Shanks \& St. John, 1994; Shanks and Johnstone, 1999). In the current experiment, changes were made to the original Nissen 
and Bullemer (1987) SRT paradigm to examine a number of issues related to learning and awareness in a SRT task. First, although researchers have questioned the adequacy of verbal reports, recognition, and generation tasks to diagnose explicit knowledge (Jackson and Jackson, 1995; Shanks \& St. John, 1994; Shanks \& Johnstone, 1999; Stadler and Roediger, 1998; Perruchet \& Amorin, 1992), little is known about whether measures derived from these tasks reflect a common explicit knowledge base in the SRT task. In order to partially fill this void, children were presented with all three tasks so that verbal awareness, recognition memory, and generation measures could be intercorrelated. Second, although explicit learning is age dependent (Siegler, 1998), Reber (1992, 1993) maintains that implicit learning is age invariant, a hypothesis that is not consistently supported in the literature. In order to further investigate age effects on sequence learning in a SRT task, third and sixth graders were used as participants. Third, Berry and Dienes (1993) and Dienes and Berry (1997) maintain that knowledge acquired through implicit learning, versus explicit learning, tends not to transfer to related tasks, (i.e., transfer specific). The transposition results obtained with children in our laboratory are consistent with the transfer specificity hypothesis (Rabinowitz and Howe, 1994; Rabinowitz and Herder, 2000). However, Willingham (1999) found that implicit knowledge acquired by adults does transfer when different perceptual stimuli and sequences are experienced in a SRT task when participants experience the same response location sequence. In order to further explore transfer specificity, bi-directional transfer was examined with children. Lastly, decreased task complexity, as defined by more redundant information in a sequence, has been associated with faster learning in a SRT task with adult populations (Stadler, 1992, 1993; Stadler \& Neely, 1997) and increased verbal awareness in a covariation task using school age children (Noseworthy, 1996). Furthermore, increased task complexity has been associated with the failure of 6 -to 8 -year-olds and 9 - to 11 - 
year-olds to implicitly learn a repeating sequence when they responded in the standard mode, i.e., bimanually, in a SRT task (De Guise \& Lassonde, 2001). In order to assess the effects of sequence structure on sequence learning and awareness in school age children, participants experienced one of three types of sequential structures varying in redundancy.

\section{Learning without awareness in an SRT task}

Although a number of researchers claim learning can occur without awareness, others have questioned the adequacy of the measures used to assess awareness (Jackson and Jackson, 1995; Shanks \& St. John, 1994; Shanks \& Johnstone, 1999; Stadler and Roediger, 1998; Perruchet \& Amorin, 1992). Willingham, Nissen, and Bullemer (Experiment 1,1989) used the Nissen and Bullemer SRT paradigm to examine learning and awareness using a university population, and assessed awareness using two measures: verbal reports and a generation task. Participants were randomly assigned to either an experimental or control group. The experimental group experienced a 10-trial repeating sequence over four 100-trial blocks. A series of questions to assess their verbal awareness of the sequential structure followed. Participants who reported not noticing any type of sequential structure and/or were unable to state three consecutive stimulus positions were classified as having no explicit knowledge; those who reported noticing the sequence and could state four to nine consecutive positions were classified as having some explicit knowledge; and participants who stated they noticed the sequence and could report all positions were said to have full explicit knowledge. Subsequently, participants in the experimental group and in an untrained control group completed two 10-trial blocks in a generation task. On each trial, they were required to predict where the next stimulus would appear by pushing the button under the expected location. Feedback 
was provided on each trial by illuminating the predicted stimulus only after the correct button was pressed.

In the SRT task, each participant's median reaction times (RTs) on successive 10trial blocks were calculated then averaged across a 100-trial block. The difference between average median RTs on the first and last 100-trial block was used as a measure of learning. In the generation task, the accuracy on the first 10-trial block was used as a measure of participants' knowledge of the 10-trial repeating sequence experienced in the SRT task, and the second 10-trial block as a measure of explicit learning. Willingham et al. (1989) reported each of the three awareness groups who experienced a repeating sequence learned more than the quasi-random control group from the original Nissen and Bullemer (1987) study. On the generation task, the accuracy of the no explicit knowledge group was similar to the accuracy of the Willingham et al. (1989) untrained control group and was significantly below the accuracy of the full explicit knowledge group on the first 10-trial block. The generation accuracies of the no explicit knowledge and the some knowledge groups were not significantly different. The authors suggested the generation data provided additional evidence that the no explicit knowledge group had learned the repeating sequence implicitly. Willingham et al. (1989) concluded two dissociable memory systems exist and that learning occurs in the absence of explicit knowledge.

In contrast, Perruchet and Amorin (1992) suggested that all RT improvements in the SRT task were a result of explicit knowledge and, therefore, could be explained by one memory system. They argued the generation task used by Willingham et al. (1989) did not provide an adequate assessment of awareness because participants were not told what sequence to generate and were provided with feedback which might have disrupted performance. Perruchet and Amorin (1992 Experiment 1) presented two 100-trial blocks 
of the same repeating 10-trial sequence used by Willingham et al. (1989) to an experimental group while a control group experienced a quasi-random sequence. Subsequently, participants were instructed to generate the previously practiced sequence without receiving feedback after each response. The analysis of the RTs in the SRT task revealed the experimental group had faster RTs than the control group by the second block of trials. A fine grain analysis of RT performance in the SRT task and accuracy in the generation task demonstrated that faster RTs on some stimulus positions were associated with better generation performance on some three and four trial runs ending in these positions. Consequently, the authors concluded explicit knowledge was responsible for the learning.

Willingham, Greeley, and Bardone (1993) attempted to rebut Perruchet and Amorin's conclusion. In their SRT task, an experimental group responded to a 16-trial repeating sequence and a control group responded to quasi-randomly displayed stimuli. Participants' verbal awareness of the repeating sequence was assessed using a series of interview questions. The authors found the experimental group's RT performance was faster on the verbally reported segments as compared to unreported segments, but RTs on unreported segments were still faster than those of the control group. In an attempt to minimize the possibility that participants acquired explicit knowledge of the repeating sequence, conservative criteria were used to categorize participants into a no explicit knowledge group. The two participants meeting these criteria apparently learned implicitly.

Meulemans et al. (1998) also claimed that SRT learning could occur without explicit knowledge based on measures of recognition memory. A group of university undergraduate students and two groups of children aged 6 to 7 and 10 to 11 experienced five 84-trial blocks of intermixed random/repeating sequences. Each block started with 
four random trials which were followed by five 16-trial sets. Each set consisted of a repeating 10 -stimulus sequence followed by six random stimuli. Following the SRT task, participants were told that the stimuli they experienced contained a repeating sequence. They were then given a recognition memory test in order to assess explicit knowledge of the repeating sequence. This task involved responding to 4-stimulus part sequences, in the same manner as in the SRT task, and judging whether the part sequence was contained in the repeating sequence. Half of the part sequences were old. The new part sequences were based on the old sequences with the exception that the last trial was quasi-randomly determined such that it was not a repetition of the third trial and created a new sequence that was not present in the original 10-stimulus sequence.

Learning in the Meulemans et al. (1998) study was assessed by calculating the difference in participants' median RTs on repeating vs. random stimuli. All participants responded faster to the repeating stimuli. The recognition ratings on the old vs new sequences did not differ significantly. The authors concluded all participants learned the sequence implicitly and claimed "... the analysis in the recognition task showed performance levels which suggest that participants did not acquire any explicit knowledge of the repeating sequence." However, Meulemans et al.'s (1998) recognition memory test may not have been sensitive enough to detect relevant traces of explicit knowledge acquired during training (Shanks \& St. John, 1994; Shanks and Johnstone, 1997) because the only cues differentiating the old and new part sequences appeared in the fourth elements. In contrast, if some new part sequences contained salient elements making them obvious sequences, i.e., ringers, then this recognition assessment may reveal participants do acquire some explicit knowledge about the sequence structure even though they might learn the complex sequences implicitly. 
Perruchet and Amorin (Experiment 2, 1992) also reported non-significant recognition rating differences between old and new 4-trial sequences. Participants experienced one block of six successive 10-trial sequences. Subsequently half the participants completed a free-generation task, described earlier in Perruchet and Amorin (1992, Experiment 1), and the others, a recognition task. The latter consisted of rating 10 different four-trial sequences based on the repeating sequence and 10 different four-trial sequences that were randomly generated with the constraint that successive trials were not repeated. Participants were instructed to respond in the same manner as in the SRT task and after each four-trial sequence rate their recognition on a four point scale. A control group experienced random sequences in the SRT task and subsequently performed the free-generation task. The authors reported participants in the repeating sequence group did not rate the old sequences significantly different from the new sequences, although this difference approached significance. However, participants in the repeating sequence group who experienced the generation task generated some part sequences more often than the control group.

In Experiment 3, Perruchet and Amorin (1992) used a dual task three choice SRT paradigm and reported a significant difference between participants' recognition scores on old and new part sequences. In the SRT task, participants responded as per Experiment 2 except the asterisk stimulus appeared in one of three locations above three response buttons during four 100-trial blocks. In addition, after each stimulus was displayed a low or high pitch tone was presented and participants were instructed to report the number of high pitch tones that occurred after each block of trials. Participants were randomly assigned to one of three groups. In the two experimental groups, two participants from each group experienced one of the following 5-trial repeating sequences over the four 100-trial blocks: A-B-C-B-C, A-C-B-C-B, B-A-C-A-C, B-C-A-C-A, C-A- 
B-A-B, C-B-A-B-A. Note that these 5-trial sequences appear to be low in complexity in comparison to those commonly used in 4-choice tasks as they consist of a unique element followed by a double alternation of the two remaining elements. Participants in the control group experienced a quasi-random sequence of stimuli. Subsequent to the SRT task, participants in one experimental group and the control group completed a freegeneration task. Participants in the other experimental group were given a recognition task similar to that described in Experiment 2 with the exception that half the old and new sequences were five- rather than four-trial sequences.

Across trial blocks, the SRTs were similar for all groups. Irrespective of this lack of evidence for learning, the authors suggested RTs on some serial positions in the structured sequences may have been longer than the RTs on the same serial positions in the random sequences and "... hence partially compensating for the improvement in performance observed on the other serial positions. An analysis of RT as a function of the serial positions was performed on the last block of trials." (p. 794). The RTs were significantly different and the authors concluded participants who experienced a repeating sequence acquired knowledge of the sequence. Also, these participants differentiated old and new sequences in the recognition task and produced partial segments of the repeating sequence in the generation task. Because the training sequences were low in complexity, it is not surprising that participants differentiated the quasi-randomly generated new part sequences in Experiment 3, but failed to do so in Experiment 2.

Using verbal reports, generation, and recognition tasks researchers have reported inconsistent conclusions regarding whether learning is explicit. However, it is likely some measures, the recognition measures in particular, lack sensitivity to aspects of the explicit knowledge of the sequential structure participants' acquire in the SRT task. 


\section{Age Invariance in Implicit Learning}

Reber $(1992,1993)$ hypothesized that hard-wired modular structures that operate independently of consciously controlled processes predate the evolutionary development of conscious awareness and that conscious awareness is built on these older structures. Based on these hypotheses, Reber $(1992,1993)$ claimed implicit learning is age invariant in contrast to explicit learning which is age dependent (Siegler, 1998). Since the early 1990s an increasing number of implicit learning studies have been conducted with children and, in particular, with infants (for an extensive review see Rovee-Collier, Hayne, \& Columbo, 2000) and children with impairments (De Guise, Jambaque, Dulac, \& Lassonde, 1999; Fletcher, Maybery, \& Bennett, 2000; Inui \& Suzuki, 1998; LaForce, Hayward, \& Cox, 2001). However, these populations are likely not the most ideal populations to investigate Reber's theory of age invariance. During infancy there are major organic structural changes that occur in the brain (Johnson, 1997). Also, learning deficits associated with brain impairments and developmental delays may also reflect structural changes in the brain which may be unrepresentative of normal development.

In the normal population, age invariance of implicit learning has been reported by the majority of researchers (Howard \& Howard, 1989; 1992; Litke, 2001; Meulemans et al. 1998; Noseworthy, 1996; Rabinowitz \& Howe, 1994; Roter, 1989, as reported in Reber, 1993; Vinter \& Perruchet, 2000; 2002). However, some researchers have found implicit learning is age dependent (Cherry \& Stadler, 1995; Curran, 1997; Howard \& Howard, 1997; 2001; Maybery, Taylor, \& O’Brien-Malone, 1995 Reber, 1967) and others have found implicit learning is age dependent in particular age ranges with children (De Guise and Lassonde, 2001; Thomas and Nelson, 2001). 
Developmental studies with adults. Howard and Howard $(1997 ; 2001)$ and Curan (1997) used embedded random stimuli in a repeating sequence and reported age related effects in a SRT task with adults. Participants in Howard and Howard (1997) responded to a repeating sequence and random trials which alternated: 1 r 4 r 3 r 2 r; r represents a randomly generated stimulus and the numbers $1-4$ represent the positions, from left to right, of the stimuli that repeat across the 8-trial blocks. There were no constraints on the random generation of stimuli and therefore a stimulus may have appeared consecutively in the same location. Two groups of participants, aged 20 and 70 years old, experienced twenty-one 90 -trial blocks. Each block started with 10 random trials which were followed by 80 trials containing the alternating repeating/random sequence. At the end of the last block, participants completed a written questionnaire in which they were asked to identify any strategies they employed while performing the task. The participants then repeated the experiment five more times on different days. At the end of the sixth day, participants were interviewed and asked a series of questions about the regularity of the repeating stimuli.

Howard and Howard (1997) found that participants did not acquire any explicit knowledge of the sequence and the younger participants learned more than the older group. In contrast to their previous age invariance results (Howard and Howard, 1989, 1992), they speculated the embedded random stimuli eliminated simple stimulus pairs (e.g., AB, CD, DA, etc.), disrupted organizational processes during learning, and placed greater demands on short term memory. Any or all of these changes might have differentially impaired the learning of older, as compared to younger, adults.

Cherry and Stadler (1995) found differences in implicit learning when comparing adults of differing ability using a SRT task. A group of university students and two groups of seniors, sampled from different populations, comprised three groups: old-low 
(low-ability older-adults with a mean age of 67.0 years); old-high (high-ability olderadults with a mean age of 67.9 years), and university students with a mean age of 22.0 years. The participants experienced an initial 100-trial block of quasi-randomly ordered stimuli and then experienced a total of eight 100 trial blocks that contained a 10-trial repeating sequence. At the end of the last block, participants were questioned about the repeating sequence and then were required to complete a generation task. The authors found all groups learned the repeating sequence in the SRT task with little verbal awareness and claimed all participants learned implicitly. They reported no significant difference in implicit learning between the young and old-high group of participants, but found a significant difference in implicit learning between these groups and the old-low group. The authors concluded ability factors affected implicit learning in older adults. On the generation task, the young and old-high groups showed similar continuous improvements in performance. The old-low group of participants showed an initial improvement during the first two cycles of the repeating sequence, but showed little evidence of further learning in subsequent trials.

Reber (1967) used letter strings, that were created according to a formal system of rules referred to as an artificial grammar, to study implicit learning. Participants first memorized letter strings based on the artificial grammar. Immediately following learning, they were informed about the grammar rules, shown new letter strings which represented both grammatical and ungrammatical strings, and were then asked to judge the grammaticality of each string. Reber (1967) reported undergraduates made more correct responses on the test trials than did high-school seniors and questioning revealed all participants knew little about the grammar (learned implicitly).

Studies with children. Maybery et al. (1995) reported age influenced both implicit and explicit learning in young children using a covariation learning paradigm. 
They varied age ( 5-7 and 10-12 year-olds) and IQ (low, medium, and high) in an implicit learning and an explicit learning task. The tasks were administered on separate days and the presentation order of the tasks was counterbalanced within each of the six groups determined by age and IQ. In the learning phase of the implicit task, the experimenter told the child that when he brought out a covered ( $4 \times 4$ matrix) board containing pictures of different objects (e.g., chair, house, shoe) to point to the picture of the house when the cover was removed. The quadrant that contained the house was determined by the color of the matrix apparatus and whether the experimenter approached the child from the left or right side when he brought out a matrix board. During the test phase, a similar process was followed except the pictures remained covered and the child was asked to guess where the house could be found. After the test phase, the child's verbal awareness of the covariation rule was assessed by asking a series of questions. Each question was more specific than the previous question. On the alternate day an explicit learning task was given. This involved the child discovering the covariation rules determined by a $3 \mathrm{x}$ 3 matrix with the experimenter identifying the salient cue dimensions for the rule discovery.

As expected, the results from the explicit learning task revealed the older children aged $10-12$ years old and the children with higher IQs outperformed the younger children aged 5 - 7 years old and lower IQ children. In the implicit learning task, none of the children reported the covariation rule. However, the older children correctly guessed the quadrants determined by the side of approach, but not the color of the apparatus, cue more often than did the younger children. Also, in contrast to the explicit task, performance was not related to IQ. Mayberry et al. claimed all the children learned this task implicitly and performance was affected by age. 
Thomas and Nelson (2001) examined sequence learning in a SRT task using 4-, 7, and 10-years-old. In Experiment 1, the 7- and 10-year-olds responded to spatial stimuli that were bitmap images of a golden retriever. The children were "... instructed to 'catch" the dog as quickly as possible by pressing the button that corresponded to the dog's spatial location." (p. 369). They were allowed to use any finger combination in responding. The authors reported most of the 7-year-olds used their index finger from each hand while the 10-year-olds tended to use two fingers from each hand. The children were randomly assigned to one of two conditions: a no-exposure condition or a preexposure condition. In the preexposure condition, children were told about the repeating sequence and observed three cycles of the sequence. In the no-exposure condition, children were not informed about the repeating sequence. The children experienced 100-trial blocks. On Blocks 1 and 4 a quasi-random sequence was displayed while a repeating sequence was used on blocks 2,3 , and 5 . After training children's awareness of the sequence was assessed through verbal reports and a generation task. "To control for baseline reaction-time differences between groups, a sequence-specific learning effect was calculated using a proportional measure of magnitude comparing the difference between random and sequence trials to overall RT for each subject (i.e., [Block4 - Block 5] / [Block 4 + Block 5]" (p. 372). There was no significant difference in learning between the two age groups. Preexposure, as compared to no-exposure, was associated with rate of learning and explicit knowledge.

In their second experiment (Thomas \& Nelson, 2001), 4-year-olds were used as participants and most of the children demonstrated implicit learning of the repeating sequence. There was no effect of preexposure and few participants acquired any explicit knowledge of the sequence structure. Using the proportional measure, the authors found 
that the 7-and 10-year-olds in Experiment 1 learned more than did the 4-year-olds in Experiment 2.

De Guise and Lassonde (2001) examined sequence learning using four groups of children: 6- to 8-year-olds, 9- to 11-year-olds, 12- to 14-year-olds, and 15- to 16-years. The authors hypothesized that the corpus callosum, "which ensures the exchange of information between the two cerebral hemispheres" (p. 253), and is relatively immature in children younger than 12 years of age, influences sequence learning in a SRT task. In order to investigate this hypothesis, children's RT performance in bimanual and unimanual responding conditions were compared. Unfortunately, the authors confounded sequence structure with these conditions. They found 6- to 8-year-olds and 9- to 11year-olds, but not the 12- to 14 year-olds and 15- to 16 -year-olds, failed to implicitly learn the sequence in the bimanual condition. In contrast, all children in the unimanual condition learned the sequence at a similar rate. The authors concluded that the most likely explanation for this interaction between age and uni-/bimanual responding was the maturation of the corpus callosum. However, since children in this age range implicitly learn less complex sequences bimanually (Meulemans et al.,1998; Thomas \& Nelson, 2001), normal adults implicitly learn sequences of equal or greater complexity (Willingham et al., 1989), and structure has been shown to influence implicit learning in adults (Stadler, 1992; Stadler \& Neely, 1997), it is possible that the complexity of the sequence, rather than the manuality of responding, accounts for the failure to learn. In summary, although the majority of researchers have found that implicit learning is age invariant, there is enough empirical evidence to question Reber's hypothesis that implicit learning is age invariant. Older adults and adults with low abilities learn at a slower rate than that of young adults (Cherry and Stadler,1995, Curan, 1997; Howard and Howard, 1997), and 6- to 8-olds and 9- to 11-year-olds fail to 
implicitly learn complex sequences (De Guise and Lassonde, 2001). It appears that, at least in some situations, age is correlated with the rate of implicit learning. Transfer Specificity of Implicitly Acquired Knowledge

While a number of authors contend that knowledge acquired through implicit learning is transfer specific (Berry \& Dienes,1993; Dienes \& Berry, 1997; Cohen, Poldrack, and Eichenbaum, 1997), inconsistent empirical findings have been reported (Altmann, Dienes, \& Goode, 1995; Perruchet \& Pacteau, 1990; Rabinowitz \& Herder, 2000; Rabinowitz and Howe, 1994; Reber, 1969; Tunney \& Altmann, 1999; Willingham et al.,1989; Willingham, 1997; Willingham, 1998a; 1998b; Willingham 2002).

Transposition studies. Rabinowitz and Howe (1994) found that implicit knowledge of the middle concept does not transfer to a novel dimension and consequently could be considered transfer specific. In Experiment 2, children in Grades $2,3,4$, and 5 were presented sets of three stimuli (e.g., dowels varying in height) and learned with feedback to pick the middle stimulus (e.g., the middle-height dowel). They were told that one of the stimuli was always correct, and the experimenter would say correct if they pointed to the correct stimulus, and the experimenter would say wrong if they pointed to an incorrect stimulus. After each child reached the training criterion of 9 correct responses in 10 successive trials, a series of transfer trials involving novel dimensions were presented. The children were told that if they thought about what they had learned, they would be able to point to the correct stimulus. No feedback was provided. After the transfer test was completed, the children were asked how they solved the problem. If they answered with middle or second, they were considered to be verbally aware. Rabinowitz and Howe (1994) found that implicit, unlike explicit, learners failed to transfer the middle concept and took more trials to reach training criterion than did the explicit learners. 
In a similar experiment, Rabinowitz and Herder (2000) investigated whether different transfer functions were associated with implicit and explicit learning. The children were trained to criterion on perceptual and conceptual dimensions and then given a transfer task using old and new dimensions. They hypothesized "Transfer in implicit learners should be associatively based and contextually bound while transfer in explicit learners should be conceptually based." Consistent with this hypothesis: (a) explicit learners were more likely to choose the middle value on new and old transfer dimensions; and (b) implicit learners were more likely to choose the stimulus closest in absolute value to the correct training stimulus on the old perceptual dimensions. Since the stimuli on the conceptual dimensions were not physically related, no consistent pattern of transfer was expected with these dimensions. Contrary to expectation, when the implicit learners were presented with pictures of a young woman, a middle-age woman, and an elderly woman during learning, and pictures of an infant, toddler, and a young boy during transfer, they responded absolutely and chose the young boy. Similarly, if the letters D, E, and F were used during training, and the letters $\mathrm{P}, \mathrm{Q}$, and R during transfer, they chose P. Among the transfer stimuli, the young boy was closest in age to the correct training stimulus, the middle-age woman, and the letter $\mathrm{P}$ was closest alphabetically to the correct training stimulus, E. Thus, the ordering information (i.e., declarative knowledge) the children acquired over several years, transferred to a laboratory task in which both learning and transfer were probably implicit.

Artificial grammar. Reber (1969) investigated transfer of implicit knowledge using an artificial grammar with university students. Letter strings of varying length that adhered to the rules of the artificial grammar were presented to participants one at a time for five sec. After presentation of three different letter strings, participants were required to write down each of the strings. If the three letter strings were correctly recalled, 
another three strings were presented. This process continued until a total of 18 strings were presented and written correctly. Participants then repeated the learning procedure with new letter strings consisting of either: different letters using the same grammar, the same letters using a new grammar, new letters using a new grammar, or no changes. Reber reported that the errors made in the transfer task increased substantially when the grammar changed but not when the letters changed and, therefore, claimed participants acquired abstract knowledge about the grammar.

Altmann et al. (1995) used an artificial grammar with university students in Experiment 4 to construct one set of strings instantiated with symbols and another set with three-letter nonsense words (referred to as syllables). The two sets of stimuli were mapped onto one another, i.e., a circle was mapped to sog, a triangle to rud, a cross-hair to kav, such that the syllable sequence sog, rud, kav also appeared as the symbol sequence circle, triangle, cross-hair. In the first phase of the experiment, an experimental group was asked to memorize sets of symbol strings of varying length that were constructed according to the artificial grammar. In a second phase, participants were informed about the artificial grammar and asked to determine whether new symbol and syllable strings were grammatical or ungrammatical. These new symbol and syllable strings contained equal numbers of legal and illegal strings. A control group also performed the second phase of the experiment without prior exposure to symbol strings. Altmann et al. found the experimental group correctly classified more syllable and symbol strings than did the control group.

Perruchet and Pacteau (1990) argued that a participant's ability to discriminate between grammatical and ungrammatical sequences in a typical grammar study, i.e., Reber (1969), does not necessarily demonstrate an abstraction of complex rules. They contend that performance can be accounted for by learning pairwise associations. In their 
Experiment 1 , university students who learned only legal letter pairs could discriminate grammatical and ungrammatical letter strings. Moreover, when the letter strings with an illegal starting letter were removed from the test set, participants who only memorized letter pairs, performed similarly to participants who learned full letter string exemplars. In Experiment 2, additional evidence inconsistent with rule abstraction was obtained. During learning, all participants were presented with letter strings to memorize. During testing, two types of ungrammatical letter strings were presented which contained either: nonpermissible pairs of letters (NP) which were two letters that could not occur together as per the artificial grammar, or nonpermissible order pairs (NO) which were permissible letter pairs that were in the wrong location in the letter string. For example, if XV was a permissible pair in the grammatical letter string RTXVC, then the string RXTVC could be considered to contain an example of a NP pair and RTCXV a NO pair. Perruchet and Pacteau found better discrimination when a NP, as compared to a NO, pair was included in the test string and contend that successful performance in artificial grammar studies usually reflects knowledge of legal letter pairs not the abstraction of a complex set of rules.

Tunney and Altmann (1999, Experiment 1), modified the procedure used by Altmann et al. (1995, Experiment 4) that was previously discussed, by eliminating sequences containing fewer than three elements during training and including test sequences containing ungrammatical strings starting with both legal and illegal elements. They found university students classified the ungrammatical strings as accurately when the test strings were from the same domain as the training domain, regardless of whether the first element in the string was legal or illegal. However, participant's ability to discriminate ungrammatical sequences composed of test strings from a domain other than the training domain was largely accounted for by the rejection of strings containing 
illegal starting elements. They hypothesized participants learned that starting elements in the grammatical sequences only occurred in this initial position and recognized illegal starting elements because they occurred elsewhere in the sequence. Tunney and Altmann investigated this hypothesis in Experiment 2 by replacing all ungrammatical test strings that contained illegal starting elements with ungrammatical strings containing legal starting elements. As in Experiment 1, participants could correctly classify ungrammatical sequences when the test strings were selected from the training domain, but were unable to discriminate ungramatical and grammatical test strings selected from a novel domain that contained legal starting elements. They concluded that participants do not broadly apply abstract grammar rules across domains in artificial grammar tasks.

SRT tasks. Willingham et al. (Experiment 3,1989) examined knowledge transfer using the same 10-trial repeating sequence presented in Experiments 1 and 2.

Participants were first given response training which consisted of teaching participants a color-response mapping of four colors to four different response buttons. This was done by displaying one of four colored rectangles in the middle of the screen and requesting participants to press the correct associated response button. The order in which the colors appeared on the screen was quasi-randomly determined and participants were required to reach a training criterion "... of three successive blocks of 100 trials with a mean RT of $600 \mathrm{~ms}$ or less and an accuracy of $90 \%$ or better" (p. 1056). Participants were then arbitrarily assigned to one of three experimental conditions: perceptual, response, or control. In all conditions, participants responded to the color of the stimulus which appeared in any one of four horizontal positions. In the perceptual condition, the positions in which the stimuli appeared followed the repeating 10-trial sequence while the colors appeared in a quasi-random sequence in which no color repeated on consecutive trials. In the response condition, the positions in which the stimuli appeared was quasi- 
randomly determined so that no stimulus was displayed consecutively in the same position while the sequence of colors was ordered so that the correct responses followed the repeating 10-trial sequence. In a control condition, both the position and color sequences were determined quasi-randomly. Subsequently, all participants were presented a transfer task in which all stimuli were white and participants were instructed to press the button below the location where the stimulus appeared. The order of stimulus positions was determined by the repeating 10-trial sequence. Following the completion of the transfer task, participants were asked if they noticed whether any of the stimuli appeared in a repeating order. If a participant answered yes then he/she was asked to indicate the stimulus pattern order. Participants were considered to have acquired some explicit knowledge if they could identify at least 4 consecutive stimulus positions in the sequence.

Based on the verbal reports, no participants in the perceptual and control conditions acquired any explicit knowledge while 14 out of 61 participants in the response group acquired some explicit knowledge of the repeating sequence. Learning was examined by comparing RTs of participants in the control, perceptual, the response with no explicit knowledge, and the response with explicit knowledge conditions. Participants with and without explicit knowledge in the response condition learned. However, it appears that knowledge did not transfer across tasks as all groups had similar RTs on the transfer task. The authors concluded that learning in the SRT task was neither purely perceptual nor motoric, but rather learning consisted of a "series of conditionaction statements mapping stimuli onto responses" (p1058).

Willingham (1999, Experiment 2) used digits "1" through " 4 " that appeared in the middle of the screen and asterisks that appeared in four boxes arranged horizontally on the screen. The use of stimuli in the middle of the screen eliminated the possibility of 
participants learning a sequence of eye movements (Mayr, 1996). During training, participants in the spatial condition experienced a sequence of asterisks and those in the digit condition experienced a sequence of numbers mapped to the four response keys from left to right. On Trial Blocks 2 to 4 , the sequence repeated and on Blocks 1 and 5 it was random. Participants in the random control condition, experienced randomly displayed digits on each trial block during training. During transfer, all participants responded to a spatial sequence of asterisks that were random on Trial Blocks 6, 7, and 9, but the original repeating sequence appeared on Trial Block 8 . Following transfer, a free generation task was used to assess awareness in all groups except those in the random digits condition. During training, participants in the spatial and sequence digit conditions, but not the random condition, decreased RTs across trial blocks and showed a large RT increase when the random stimuli were presented on Trial Block 5. In both the spatial and sequence digits conditions, but not the random condition, RTs significantly decreased on the third block of transfer trials when the repeating sequence was presented. This finding was robust even when a stringent criteria was applied to eliminate participants from the analysis who may have acquired explicit knowledge. The author concluded these results demonstrate implicit knowledge is not transfer specific and learning in a SRT task is not solely perceptual nor does it depend on "stimulus-response pairings" (p. 566) as previously hypothesized (Willingham et al., 1989).

In the third experiment (Willingham, 1999), implicit sequence knowledge was also found to be flexible. The procedure was similar to that used in Experiment 2 with the following exceptions. During SRT training, only spatial stimuli, i.e., asterisks, were used and participants in perceptual and motor conditions experienced an altered stimulus-response-button mapping. Participants in the spatial and motor conditions were instructed not to press the response button directly under the asterisk but to press the 
button that was one position to the right. Participants in a SRT condition experienced the standard mapping during both training and transfer. During transfer, participants in the perceptual and motor conditions experienced the same sequence, either perceptual or motor, respectively, as in training and the standard SRT stimulus-response-button mapping. Therefore, participants in the motor and SRT conditions experienced the same sequence of response locations during both training and transfer. The results reflect only participants in the motor and SRT conditions showed significant learning during transfer. Willingham concluded that results from these experiments demonstrate the flexibility of knowledge acquired through implicit learning in a SRT task.

In Willingham et al. (2000, Experiment 2), the authors investigated the effect of transfer by disrupting motor sequence movements. In the Fingers, Locations, and Random conditions, participants responded during training with their hands crossed and during transfer with their hands uncrossed. The stimulus sequence and, thus, the response locations changed in the Fingers condition during transfer so that the same sequence of finger movements was used in training and transfer. Participants in the Locations condition experienced the same stimulus sequence during training and transfer and, thus, the response locations did not change but a different sequence of finger movements was required during transfer. In the random condition, participants experienced a random sequence on every block except on the third block during transfer which provided a control for learning on transfer. In a fourth condition, referred to as the Both condition, participants responded using the standard SRT task in both training and transfer.

Knowledge acquired by participants in the Locations condition transferred from training to the transfer task when participants responded with a different finger sequence which is consistent with a number of studies that report SRT learning is effectorindependent (Cohen et al., 1990; Keele, Jennings, Jones, Caulton, \& Cohen, 1995; 
Japikse, Negash, Howard, \& Howard, 2003). In contrast, participants in the Finger condition did not appear to learn any more about the sequence during transfer than those in the random condition. Therefore, changing the response locations disrupted transfer. The authors concluded "that the sequence of response locations must be retained in order for implicit sequence knowledge to transfer" (p. 372).

Based on the literature review, it appears implicitly acquired knowledge by children in transposition studies (Rabinowitz \& Howe, 1994; Rabinowitz \& Herder, 2000 ) and by adults in artificial grammar studies is transfer specific (Perruchet \& Pacteau, 1990; Tunney \& Altmann, 1999). However, the specificity of transfer of implicit knowledge acquired by adults in a SRT task is more difficult to characterize. Although it is clear that transfer of implicit knowledge is effector independent (Cohen et al., 1990; Keele, Jennings, Jones, Caulton, \& Cohen, 1995; Japikse, Negash, Howard, \& Howard, 2003; Willingham et al., 2000), it is not clear when transfer will occur when participants experience sequences instantiated with different stimuli associated with the same response sequence. In Willingham et al. (1989) participants' knowledge acquired with a stimulus sequence of colors failed to transfer to a stimulus sequence of asterisks. However, in Willingham (1999, Experiment 2), adults transferred implicit sequence knowledge acquired with a sequence of digits to a sequence of asterisks.

\section{Task Complexity}

When repeating sequences of the same length are presented, sequence structure has been found to affect adult participants' SRTs (Stadler, 1992; Stadler \& Neely, 1997). As sequence structure increases there are fewer unique runs and specific runs occur more often. For example, the sequence $\mathrm{ABCABCD}$ is more structured and less complex than the sequence $\mathrm{ABCBDAC}$. The former contains only three unique 2-trial runs $(\mathrm{CA}, \mathrm{CD}$, $\mathrm{DA})$, and three runs which repeat twice $(\mathrm{AB}, \mathrm{BC}, \mathrm{ABC})$, whereas the latter contains 
seven unique 2-trial runs $(\mathrm{CA}, \mathrm{AB}, \mathrm{BC}, \mathrm{CB}, \mathrm{BD}, \mathrm{DA}, \mathrm{AC})$ and no runs that repeat. Stadler (1992) used three sequences to investigate the effects of statistical structure on RT performance. University students experienced eight 100-trial blocks. The first seven blocks consisted of 10 repetitions of a 10-trial repeating sequence and the last block consisted of quasi-randomly displayed stimuli. Sequence learning was measured by the RT decrease across Trial Blocks 1 to 7 and the RT increase across Trial Blocks 7 and 8 . Verbal awareness was not assessed. Stadler found that the rate of sequence learning increased with statistical structure. He suggested that more practice occurred with repeating runs of stimuli which likely accounted for the faster RTs associated with the more structured, less complex, sequence.

In a later experiment (Stadler \& Neely, 1997), explicit knowledge was assessed using a recognition task. Participants were presented with a questionnaire containing four different 10-trial sequences depicted in columns and were asked to pick the repeating sequence experienced in the SRT task. One sequence was previously experienced while the other three were quasi-randomly generated. Stadler and Neely replicated their 1992 findings, but RT performance was not related to how participants answered the recognition question.

Noseworthy (1996) and De Guise and Lassonde (2001) studied the effect of task complexity on children's performance. In a covariation task, Noseworthy found task complexity was inversely related to verbal awareness of the covariation rule. In a SRT task, De Guise and Lassonde (2001), reported that 6- to 8-year-olds and 9- to 11-yearolds learned the $\mathrm{BABDCACBDC}$ sequence while responding unimanually, but failed to learn the $\mathrm{DBCACBDCBA}$ sequence when they responded bimanually. The former sequence is more structured than the latter because it contains four unique 2-trial runs $(\mathrm{AB}, \mathrm{AC}, \mathrm{BA}, \mathrm{CA})$ and three 2-trial $(\mathrm{BD}, \mathrm{CB}, \mathrm{DC})$ and one 3-trial run $(\mathrm{BDC})$ that repeats 
twice, while the latter sequence contains eight unique 2-trial runs $(\mathrm{AC}, \mathrm{AD}, \mathrm{BA}, \mathrm{BC}, \mathrm{BD}$, $\mathrm{CA}, \mathrm{DB}, \mathrm{DC})$, and only one 2-trial run that repeats twice (CB). Therefore, since the more complex sequence structure was associated with bimanual, as compared to unimanual responding, and increased sequence structure has been associated with slower SRTs with adults (Stadler, 1992; Stadler \& Neely, 1997), it is likely that sequence structure complexity, not mode of responding, accounted for the failure of 6- to 8-year-olds and 9to 11 -year-olds to learn the repeating sequence.

\section{Experimental Objectives}

A number of the issues discussed above were investigated using an SRT task with children. First, three tasks were used to assess explicit knowledge: verbal reports, recognition, and generation. If measures derived from these tasks reflect a common explicit knowledge base acquired during training, intercorrelations between these measures should be consistent. In addition, it was suspected that the measures derived from the recognition task used by Meulemans et al. (1998) may not be sensitive to some explicit knowledge, i.e., obvious part sequences. To increase the sensitivity of this task, the ringer sequences, i.e., $\mathrm{ABCD}, \mathrm{AABB}, \mathrm{ABAA}, \mathrm{AAAB}$, were included to determine whether participants explicitly notice the absence of simple patterns while they implicitly learn complex patterns. It was expected that only the ringers would be correctly differentiated on the recognition test because explicit knowledge of the repeating sequence might not be sufficiently robust to differentiate old and new complex sequences.

Second, to examine the effects of age on learning and awareness in a SRT task, third and sixth graders were used. Since older children learn explicit tasks faster than younger children (Siegler, 1998), if SRT learning is explicit or partially explicit then: (a) the learning curves of the older, as compared to the younger, children should be steeper sloped reflecting faster acquisition rates; (b) a higher percentage of older, as compared to younger, children 
should report awareness or partial awareness of the sequential structure; and (c) older children should make fewer recognition and generation errors than younger children (Willingham et al., 1989, 1993).

Third, sequence structure was varied to examine the effect of task complexity on children's implicit learning and awareness in a SRT task. Sequence structure has been reported to affect implicit SRT learning in adults (Stadler, 1992; Stadler \& Neely, 1997) and is suspected to have affected implicit SRT learning in children (De Guise \& Lassonde, 2001). Using a covariation task, Noseworthy (1996) found that as task complexity increased the percentage of children who expressed verbal awareness decreased. To the extent that task complexity in a covariation task is related to sequence structure in a SRT task, sequence learning and verbal awareness was expected to correlate with sequential structure in a SRT task.

Finally, Berry and Dienes (1993) and Dienes and Berry (1997) claim that one of the primary characteristics of implicit knowledge is that knowledge acquired in one task does not readily transfer to other related tasks (transfer specificity). Results from a number of implicit learning studies are consistent with this hypothesis (Meulemans et al., 1998; Rabinowitz and Howe, 1994; Rabinowitz and Herder, 2000; Reber 1969; Tunney \& Atmann, 1999; Perruchet \& Amorin, 1992; Willingham et al., 1989). However, knowledge acquired by adults in SRT studies appears to be flexible when participants experience the same sequence of response locations in similar tasks (Willingham, 1999; Willingham et al, 2000). In the present experiment, transfer specificity of children's implicit knowledge was examined using spatial and perceptual stimuli which were mapped to the same sequence of response locations. Participants were presented with two stimulus types, letters that were centrally located and asterisks that appeared in four horizontal locations. 


\section{Method}

\section{Participants}

The participants were 176 children who attended one of three middle-class schools located in predominately, caucasian neighborhoods. The children, their parents, and school board officials all consented to having the children participate. There were 44 male and 44 female third graders $(\underline{\mathrm{M}}=8.6$ years, $\underline{\mathrm{SD}}=0.53)$, and 44 male and 44 female sixth graders $(\underline{M}=11.3$ years, $\underline{\mathrm{SD}}=1.37)$. Due to equipment malfunction, the data files from 13 additional children were not used. In general, the conditions to which these 13 children were assigned are representative of the experimental design.

\section{Apparatus and stimuli}

An IBM computer with a 21.5 by $16 \mathrm{~cm}$ screen was used to generate the stimuli and record responses. A separate box ( $22 \mathrm{~cm}$ wide $\times 4 \mathrm{~cm}$ deep $\times 2 \mathrm{~cm}$ high) was located in front of the monitor. Four square 1.5 X $1.5 \mathrm{~cm}$ buttons, labeled "A", "B", "C", and "D" from left to right, were mounted on the top face of the box, spaced $5 \mathrm{~cm}$ apart, with $3.5 \mathrm{~cm}$ on each end. In the SRT, recognition, and generation tasks two types of stimuli were used. Either an asterisk appeared in one of four horizontal locations or a letter $(A, B, C$, or D) appeared in the middle of the screen. Children responded to the stimuli by pressing one of the four buttons on the box which were located below the four possible positions of the asterisks.

\section{Design}

The children were quasi-randomly assigned to one of 24 cells determined by the factorial combination of age (third vs. sixth graders), gender, sequential structure (high vs. moderate vs. quasi-random), and presentation sequence (asterisks presented before letters vs. letters presented before asterisks). A summary of the experimental design appears in Table 1. Eight children were assigned to each of the high and moderate structure cells while 
six children were assigned to each of the low structure cells. More children were assigned to the high and moderate structure cells because, as opposed to the quasi-random sequence, it was possible to learn these sequences and verbal awareness might correlate with performance. Children in the moderate and high structure cells experienced two sets of SRT tasks, two sets of verbal awareness questions, two recognition memory tasks, and two generation tasks. Only SRT and awareness tasks were presented to the children in the quasi-random structure condition because the recognition test sets were inappropriate and Willingham et al. (1989) found no difference in generation task performance between participants in a quasi-random structure group and those in a repeating sequence group who were verbally unaware of the sequence structure.

Insert Table 1 about here

Procedure

Prior to the beginning of the experiment, the experimenter entered the child's name, an assigned subject number, birth date, grade, gender, and condition into the computer. In all tasks, the computer program tracked and coded every stimulus presented, the associated response, and response latency.

SRT tasks. Each of the SRT tasks consisted of 320 trials presented in four, 80-trial blocks. After the child was seated comfortably in front of the computer, the following instructions, similar to those used by Meulemans et al. (1998), appeared on the screen and were read to each child who experienced asterisks in the first SRT task. The task and instructions were appropriately modified for the children who experienced the letters in the first task. In both SRT tasks, a stimulus appeared on the screen that was terminated by a correct response. The next stimulus appeared after a $250 \mathrm{~ms}$ delay. 
On the screen in front of you a little star will appear in one of these four places (experimenter pointed to the locations of where asterisks would appear). You will have to push the button that is underneath the star as soon as the star appears. If you make a mistake the computer will make a beep. This is not serious, you simply push on the correct button, and then the next star will appear. You must answer as quickly as possible, but try not to make mistakes. You will do the task for about two minutes and then you will have a little break. Dots will go across the bottom of the screen and when they reach the other side, the computer will beep twice. When you hear the second beep you can press any button to start again. This will happen three times and then you will see the word "STOP" in the middle of the screen. It means that we are ready to start the next task. Do you have any questions? Please press one of the buttons to begin.

After the child completed the first SRT task, instructions for the second SRT task appeared. This task will now be a little different. Instead of a star appearing on the screen in different places, a letter will appear on the middle of the screen. The letter will either be an "A", "B", "C", or "D". You will have to press the button with the same letter as the one on the screen. You need to do the same thing as you did in the last task: answer as quickly as possible, but try not to make mistakes. Do you have any questions? Press one of the buttons to begin.

Verbal awareness tasks. After the child completed the SRT tasks, verbal awareness was assessed using the following instructions:

I am going to ask you a couple of questions about what you just did. I don't want you to press any buttons, just tell me what you think. In the first task you pressed the button that appeared under a star. For some of the children the stars appeared in a regular order and they could learn where the next star would appear. For other 
children, the stars did not appear in a regular order and they could only guess where the next star would appear. Do you think you could tell where the next star would appear or did you guess?

Similarly, in the second task you pressed the button with the same letter that appeared on the middle of the screen. For some of the children the letters appeared in a regular order and they could learn what the next letter would be. For other children, the letters did not appear in a regular order and they could only guess what the next letter would be. Do you think you could tell what the next letter would be or did you guess?

The experimenter entered the participants' responses as either 1,2,3, or 4 corresponding to the responses, "I knew", "Sometimes knew", I didn’t know", or "I was guessing" that were displayed on the screen following each question. The latter two categories were offered to make the judgements easier for the children. Both were assumed to reflect an absence of explicit knowledge. There was no a postori evidence to indicate otherwise, so they were combined into a single data entry of " 3 ".

Recognition tasks. Following the awareness tasks, children who experienced repeating sequences in the SRT tasks were presented recognition tasks that were similar to tasks used by Meulemans et al. (1998).

The stars you saw before followed an order that you might have learned. You might remember the entire order or parts of the order in which the stars appeared on the screen. We are going to do the same thing as before: a star will appear in one of the four locations on the screen and you will press the button underneath the star. But this time you don't have to worry about pushing the buttons as quickly as you can and only four stars will appear on the screen. After you see all four stars and press the correct buttons, I am going to ask you whether you remember "Seeing this order 
of four stars before", "Are not sure whether or not you saw this order before, or "You are sure that you did not see this order before". You can see the four star order as many times as you like before you make up your mind. Just let me know if you want it repeated. When you have your mind made up, tell me what it is. Do you have any questions? Are you ready?

The experimenter pressed a key to start each four-trial sequence. After the child responded to each four-trial sequence, the above three answers were displayed on the screen in the above order. The children were asked to indicate whether each of the four-stimulus sequences presented was either:

1. An order you are sure you saw before;

2. An order you are not sure whether or not you saw before;

3. An order you are sure you did not see before.

Following the child's choice, the experimenter pressed a number from 1 to 3 corresponding to each respective answer. Following the completion of 12 four-stimulus sequences based on the asterisks, the child was read modified instructions for letters.

Generation tasks. After the recognition tasks, children who experienced repeating sequences with asterisks first were presented the following instructions:

As I told you a little while ago, the stars appeared in a way that you might have learned where the next star was going to be. For this task, one star is going to appear on the screen and instead of pressing the button under the star, I want you to press the button where you think the next star will appear. The computer will beep if you make a mistake. This is not serious. The next star will not appear until you press the correct button. Keep doing this until the word "STOP" appears on the middle of the screen.

Following thirty-nine prediction trials, participants were instructed on the letter stimuli. 
As I told you before, the letters that you saw earlier appeared in a way that you might have learned what the next letter would be. Now we are going to do the same thing except you will see one letter on the middle of the screen and I want you to press the button with the same letter you think will appear next. Keep doing this until the word "STOP" appears in the middle of the screen.

\section{Sequence structure}

Stimulus response sequences were created with the numbers $1,2,3$, and 4 which were mapped to the four positions at which an asterisk could appear and the letters A, B, C, and D. The sequence 2-4-2-3-1-2-4-1-2-4 was used in the moderate structure condition and 2-4-2-3-1-2-4-2-3-1 was used in the high structure condition. In the quasi-random structure condition, the stimuli appeared randomly with the exception that they did not repeat on successive trials. Given a repeating sequence of fixed length, sequences with increasing structure contain fewer unique runs and specific runs repeat more often (Stadler, 1992). The moderate structure condition contained three unique 2-trial runs (2-3, 3-1, 4-1), three runs which repeated twice $(4-2,1-2,1-2-4)$, and one run which repeated three times (2-4). The high structure sequence contained a repetition of the first five stimuli in the moderate structure sequence. Thus, there were no unique runs in the 10-trial high structure sequence and a large number of runs repeated twice.

Each child, except those assigned to the quasi-random structure group, experienced the same repeating 10-trial response sequence with both asterisks and letters in the SRT and generation tasks. Four variants of both the moderate and high structure sequences were employed. The particular variant a child experienced was randomly determined. The variants were generated by using four mappings. The identity mapping resulted in no change in the specified sequences (i.e., 1 to 1,2 to 2,3 to 3,4 to 4 ). Thus, a child in the moderate condition who experienced the identity mapping first saw an asterisk in the second 
position, followed by the fourth position, followed by the second position, etc., in one task; and the letter B, followed by the letter D, followed by the letter B, etc., in the other task. The remaining mappings were rotations ( 1 to 2,2 to 3,3 to 4,4 to 1 ), ( 1 to 3,2 to 4,3 to 1 , 4 to 2 ), and (1 to 4,2 to 1,3 to 2,4 to 3 ). A child in the moderate condition who experienced the last mapping described above, first saw an asterisk in the first position, followed by the third position, followed by the first position, followed by the second position, etc, in one task; and the letter $\mathrm{A}$, followed by the letter $\mathrm{C}$, followed by the letter $\mathrm{A}$, followed by the letter B, etc., in the other task.

$\underline{\text { Recognition Stimuli }}$

In each recognition test, 12 four-stimulus sequences were presented: 4 old, 4 new, and 4 ringers. The old sequences were experienced in the SRT task; the new sequences were similar to the recognition segments used by Meulemans et al. (1998) and; the ringer sequences had an obvious structure that was not previously experienced and were included to increase the sensitivity of the assessment measure to participants' explicit knowledge. The particular stimuli used and the order in which they appeared was randomly determined for each child, subject to the constraints described below. In the high structure condition, four different old sequences were randomly selected from a five sequence set which contained all the 4-trial sequences that can be derived from the high structure sequence. In the moderate structure condition, eight of the ten sequences that could be derived from the moderate structure sequence were used because two sequences were consider to be ringers, i.e., 2-4-2-4 and 4-2-4-2. The ringer sequences contained one double alternation sequence (e.g., 1-2-1-2), one double repetition sequence (e.g., 3-3-2-2), one sequence in which the same stimulus was presented three times (e.g., 2-4-2-2), and one perfectly ordered sequence (e.g., 1-2-3-4 or 4-3-2-1). To create new sequences for the high and moderate structure conditions, sequences were randomly selected from the high and moderate old sequence 
sets, respectively, and the last trial was quasi-randomly determined such that the stimulus selected was not a repetition of the third element, created a new sequence, and was not a ringer. Thus, the old sequence $2-4-2-3$ would become $2-4-2-1$.

Results

Two sets of analyses of variance (ANOVAs), referred to as general and awareness analyses, were conducted. In the general analyses, the effects of the independent variables on performance on each of the four tasks, SRT, verbal reports, recognition, and generation, were examined. In the awareness analyses, the associations of verbal awareness with the other dependent variables on each task were assessed and only significant contrast effects involving verbal awareness are reported. Since it was not possible for the children to correctly report being aware or partially aware of a quasi-random sequence, only the data obtained with children in the moderate and high structure groups were used in the awareness analyses. Although the scale of measurement for both the awareness and recognition data are ordinal rather than interval, the use of parametric statistical tests, as opposed to relying solely on nonparametric statistical tests, was considered appropriate (for a review, see Zumbo and Zimmerman,1993). Because a large number of dependent variables were examined across the two sets of analyses, an attempt was made to moderate the expermentwise error rate by setting the significance level at $.001(.01)$ for within-subject (between-subjects) contrast effects. The more restrictive significance level used with within-subject, as compared to between-subjects, effects reflects the greater statistical power associated with the within-subject tests. The Scheffé (1959) procedure was used in all follow-up tests.

The between-subjects variables in the general analyses were gender, grade (grade 3 vs. grade 6), sequential structure (quasi-random vs. moderate vs. high; the quasi-random condition was not included in the analyses of the recognition, generation, and the awareness 
data), and stimulus-type sequence (letters-asterisks vs. asterisks-letters). Depending on the dependent variable, the within-subject variables were stimulus type (letters vs. asterisks) and either trial blocks (Trial Block 1 to 4 ) or recognition sequence (old vs. new vs. ringers).

The original plan was to add verbal awareness as an additional between-subjects factor to the variables included in the general analyses. Two outcomes necessitated a change in strategy. First, separate awareness analyses had to be conducted on asterisk and letter data because some participants reported different levels of awareness with each stimulus type. Second, level of reported awareness depended on sequential structure. As a consequence, when awareness was added as a between-subjects factor some of the cells determined by combinations of the other between-subjects factors with structure and awareness were empty and others had low numbers of participants. In order to increase the stability of the awareness ANOVAs, only variables that were associated with significant contrasts in the general analyses or those of particular interest were included. For example, age only was included as a variable in the latency analyses because the older children responded faster than the younger children on all the reaction time measures. Age did not significantly affect performance with the remaining dependent variables in the general analyses.

\section{Verbal Reports.}

The verbal awareness responses "I knew" (1), "I sometimes knew" (2), and "I didn't know or I was guessing"(3), served as the dependent variable in the ANOVA. Only the main effect of sequential structure, $F(2,152)=39.59$, was significant. The mean verbal awareness ratings for the high, moderate, and quasi-random structure conditions were, 1.42, 2.13 , and 2.38 , respectively. The mean verbal awareness rating for the high structure group was significantly lower than the average of the mean rating of the quasi-random and moderate structure groups, $\underline{S}^{2}(2,128)=77.13$, while the mean ratings of the latter two 
groups did not differ significantly, $\underline{\underline{S}}^{2}(2,128)=4.77$. These findings reflect that children were more likely to report being aware with the least complex sequence. This was expected based on the data reported by Noseworthy (1996) using a covariation task. She found children were more likely to report awareness of the covariation rule when they were trained on a less complex, as compared to a more complex, training task.

Insert Table 2 about here

However, the similarity of verbal awareness ratings by children who experienced the moderate and quasi-random sequences was unexpected. Therefore, the related frequency distributions were examined. The number of children who reported being aware, partially aware, and unaware of the sequential structure in each SRT task appear in Table 2. As can be seen in the table, reported awareness was not independent of stimulus structure, $\chi^{2} s(4)>$ $38, \mathfrak{p}<.001$, as children in the high structure group were more likely to report being aware of both letter and asterisk sequences than were children in the quasi-random and moderate groups. The verbal awareness distributions of children in the two latter groups were similar, $\chi^{2} s(2)<5, p>.05$. Moreover, more than $40 \%$ of the children presented quasi-random sequences reported being aware or being partially aware of a repeating sequence. Jackson and Jackson (1995) reported a similar finding based on unpublished data. They found "that subjects trained entirely under random conditions, frequently claimed to have noticed a repeating pattern."

\section{SRT Tasks}

\section{SRT latency}

For each participant, the median RT of correct responses was calculated for each of the four 80-trial blocks and these median latency scores served as the dependent variable. 
General analysis. The older children $(\underline{\mathrm{M}}=0.60 \mathrm{~s})$ responded faster than the younger children $(\underline{\mathrm{M}}=0.88 \mathrm{~s}), \mathrm{F}(1,152)=119.99$, consistent with the results reported by Meulemans et al. (1998). Of greater interest are the slopes of the RT curves of third and sixth graders across trial blocks. As can be seen in Figure 1, the curves were approximately parallel. The failure of the age by trial block interaction to reach significance, $\underline{E}(3,456)=2.49, p>.05$, is consistent with this observation. If the decrement in RT across trial blocks reflects learning, as suggested by Willingham et al. (1989), then both age groups learned at a similar rate. Since explicit learning rates in children are age dependent (Seigler, 1998), most of the children probably learned implicitly. In general, the SRT data appears to be consistent with Reber's $(1992 ; 1993)$ assertion that implicit learning is age invariant.

Insert Figure 1 about here

The mean median-latency scores for the quasi-random, moderate, and high structure groups across trial blocks appear in Figure 2. Inspection of the figure reveals that the RTs of participants in the high structure group decreased, while the RTs of participants in the moderate and quasi-random groups remained relatively stable resulting in a significant Structure by TB interaction, $\underline{\mathrm{E}}(6,456)=19.63$. Thus, the main effect of trial blocks, $\underline{\mathrm{F}}(3$, $456)=29.09$, primarily reflects the performance of children in the high structure group. The RTs across trial blocks for each of the structure groups did not overlap. Therefore, the main effect of structure, $\underline{F}(2,152)=25.43$, is interpretable. As expected, learning rates increased with increased sequential structure (Stadler, 1992, 1997) but the difference between the mean RTs in the quasi-random and moderate structure groups was not significant, $\underline{S}^{2}(2,152)=6.52$, while the difference between the mean RT score of the high structure group and the average of the means of the quasi-random and moderate groups, $\underline{\mathrm{S}}^{2}$ 
$(2,152)=48.22$, was significant. Because the third and sixth graders did not learn the complex sequences, it is likely that sequence complexity accounts for De Guise and Lassonde's (2001) failure to find learning in 6- to 8-year-olds and 9- to 11-year-olds when they responded bimanually, as opposed to unimanually.

Insert Figure 2 about here

As can be seen in Table 3, participants in the quasi-random group who experienced letters second responded slower with the letter stimuli than did participants who experienced letters first. In every other comparison, participants who experienced a particular stimulus set second, as compared to first, responded faster to that set. The consistency of the order effect across the remaining five combinations was reflected in the Stimulus sequence $\mathrm{X}$ Stimulus type interaction, $\mathrm{E}(1,152)=30.03$. It appears that these data reflect nonspecific transfer, perhaps a warm up effect, and not transfer of sequence knowledge across stimulus types because the order effect was not restricted to children who learned in the high structure condition. It is not clear why the aberation occurred with letters in the quasirandom group, but it was associated with a triple interaction of stimulus type, stimulus sequence, and structure, $\mathrm{E}(2,152)=8.80$. Not surprisingly, further inspection of Table 3 reveals that participants responded faster to asterisks than letters, $\mathrm{E}(1,152)=110.51$, as it required less time to press the response button directly below an asterisk stimulus than to find and press the correctly labelled button when a letter stimulus appeared in the middle of the screen. Willingham (1999) reported a similar effect using centrally presented digits.

Insert Table 3 about here 
Awareness analyses. Reported awareness was correlated with response latency with letters (aware, partial, and unaware; $\underline{\mathrm{M} s}=0.69,0.80$, and $0.87 \mathrm{~s}$, respectively), $\underline{\mathrm{F}}(2,115)=$ 5.05). The mean latencies of the partial and unaware groups did not significantly differ, $\underline{S}^{2}(2,116)=1.46$, but the mean latency of the aware group was significantly faster than the average of the mean latencies of both the partial and unaware groups, $\underline{S}^{2}(2,116)=16.56$. No significant differences in latency were found between the awareness groups when the participants experienced asterisks, $\underline{F}(2,116)=1.99, p>.05$. However, the pattern represented by the mean latency of the aware, partial, and unaware groups with the asterisk data $(\underline{\mathrm{Ms}}=0.61,0.67$, and $0.69 \mathrm{~s}$, respectively) was similar to the pattern with the letter data. The association of awareness with RTs is consistent with other reported findings (Willingham et al., 1989; Curran and Keele, 1993; Mayr, 1996). The more robust relationship obtained with the letters may reflect that the letter sequences were easier to verbally code.

\section{SRT errors}

General analysis. As can be seen in Table 4, accuracy of responding was high throughout the SRT task as the average number of errors per 80-trial block was less than 4 . The number of errors across trial blocks monotonically increased with asterisks while they were relatively stable with letters, $\underline{\mathrm{F}}(3,456)=6.19$. It is likely that more anticipatory errors were associated with the faster RTs with the asterisks stimuli and this probably caused the observed interaction.

Insert Table 4 about here

Awareness analyses. No significant contrasts involving verbal awareness were obtained. 


\section{Recognition Tasks}

\section{$\underline{\text { Recognition judgments }}$}

Children's recognition judgments "I am sure I saw this order before", "I am not sure whether or not I saw this order before", and "I am sure that I did not see this order before" were scored 1,2 , and 3 , respectively, and served as the dependent variable for participants in the high and moderate structure conditions.

General analysis. Participants clearly differentiated the old, new, and ringer sequences $(\underline{\mathrm{Ms}}=1.78,2.20,2.73$, respectively), $\underline{\mathrm{F}}(2,224)=114.32)$. All pairwise follow-up comparisons were significant, $\underline{S}^{2} s(2,224)>42.78$. It is not clear why participants were more likely to judge letter sequences, than asterisks sequences, as new $(\underline{\mathrm{M}} \mathrm{s}=2.32$ and 2.15 , respectively), $\mathrm{E}(1,112)=23.45)$. It should be noted that structure did not affect recognition performance, replicating the findings reported by Stadler and Neely (1997).

Insert Table 5 about here

Awareness analyses. Although the main effects of recognition sequence, $\underline{F} s(2,244)$ $>43.50$, and awareness, $\underline{F} \mathrm{~s}(2,122)>7.82$, were significant, the recognition sequence by awareness interactions approached significance in both analyses (letters: $\operatorname{Es}(4,244)=4.08$, $\underline{\mathrm{p}}=0.003$; asterisks: $\underline{\mathrm{F}} \mathrm{s}(4,244)=3.28, \underline{\mathrm{p}}=0.012)$ and warranted further investigation. The relevant data appear in Table 5. The simple effect of the recognition sequence was significant with both stimulus types at each level of awareness, $\underline{F}(2,224)>21.49$, with the exception that the effect only approached significance in the unaware condition with the letter data, $\underline{E}(2,224)=6.26, \underline{p}<0.005$. The verbally aware participants discriminated the old and new sequences, $\underline{S}^{2} S(2,224)>20.81$, while the partially aware and unaware failed to recognize these sequences as different, $\underline{S}^{2} s(2,224)<6.46, \underline{\text { p. }}>.05$. All participants 
discriminated the old and ringer sequences; the pairwise differences were significant in all comparisons, $\underline{\mathrm{S}}^{2} \mathrm{~s}(2,224)>33.13$, except in the unaware condition with the letter data which approached significance, $\underline{\mathrm{S}}^{2}(2,224)=11.85, \underline{p}<0.005$. The children's sensitivity to the ringer sequences was highlighted by the fact that the children were more likely to judge the ringers, compared to the new sequences, as not having been experienced before in the SRT task even though both types of sequences were not previously experienced. This difference approached significance with the aware conditions and the unaware conditions with letters, $\underline{\mathrm{S}}^{2} \mathrm{~s}(2,224)>11.17, \underline{\mathrm{p}}<0.005$, but was significant in the remaining conditions, $\underline{\mathrm{S}}^{2} \mathrm{~s}(2,224)$ $>28.88$.

It was expected that children would only differentiate the ringer part sequences as Meulemans et al. (1998) reported children and adults failed to differentiate old and new part sequences in their SRT study. However, the sequence used in the Meulemans et al. study was more complex than either of the repeating sequences used here and Meulemans et al. did not assess verbal awareness which was found to influence recognition performance in the current experiement.

Recognition judgement latencies

The mean judgement latencies (average median latency between the last response to the four trial part sequence and the rating) served as the dependent variable and was a measure of sequence recognition speed.

General analysis. Participants' mean judgement latencies on the ringer, old, and new sequences were $2.24,2.93,2.96 \mathrm{sec}$, respectively, $\underline{E}(2,224)=31.83$. The faster RTs associated with the ringer sequences likely reflects that they were quickly identified as not having been experienced before. The difference in mean judgement latencies between the old and new sequences was not significant, $\underline{\mathrm{S}}^{2}(2,224)=0.09$, but the mean judgement latency with the ringers was significantly faster than the average of the judgement latencies 
with the old and new sequences, $\underline{S}^{2}(2,224)=63.57$.

Awareness analyses. No significant contrast effects involving awareness were obtained

$\underline{\text { Recognition judgement matching errors }}$

A matching error occurred when a participant pressed the wrong button in response to the displayed stimulus. The number of matching errors made with each sequence was used as a measure of transfer from the SRT task to the 4-trial recognition context.

General analysis. The number of matching errors made by participants depended on the type of sequence they experienced $(\underline{M} s=0.28,0.45$, and 0.55 for the old, new, and ringer sequences, respectively), thus, reflecting transfer, $\underline{F}(2,224)=10.99$. The mean number of matching errors with the new and ringer sequences did not significantly differ, $\underline{S}^{2}$ $(2,224)=3.22, \mathrm{p}<0.05$, but the mean number of errors collapsed across these groups was significantly greater than the mean errors obtained with the old sequences, $\underline{S}^{2}(2,128)=$ 18.78 .

Awareness analyses. No significant awareness contrast effects were obtained.

\section{Generation Tasks}

Performance on the first 10 trials of the generation task was a fairly direct measure of transfer of sequential information from the SRT task. Consequently, in both the general and awareness analyses, the dependent variable of primary interest was the number of errors participants made on the first 10-trial block. Of lesser interest was the number of errors participants made on the remaining three 10-trial blocks because both the feedback which followed each generation response and potential transfer of information acquired in the SRT task could influence performance. The latter measure was included to facilitate comparisons with data reported in other studies (Nissen \& Bullemer, 1987; Willingham et al., 1989). 


\section{Generation errors}

General analysis. The effect of structure on generation task performance has not been studied before and was found to affect performance in the current experiment. Participants in the high structure group made fewer errors than those in the moderate structure group on both dependent variables, $\underline{F} s(1,112)>16.91$, reflecting transfer from the SRT task to the generation task. The relevant mean errors for trial block 1 (mean errors collapsed across all trial blocks) were 5.07 and 7.90 (3.54 and 7.45), respectively. Consistent with findings reported by Nissen and Bullemer (1987) and Willingham et al., (1989), performance improved with practice (trial blocks 1 to $4 \underline{\mathrm{M}} \mathrm{s}=6.48,5.44,5.22,4.93$, respectively), $\mathrm{E}(3,336)=15.07$.

Insert Table 6 about here

Awareness analyses. As shown in Table 6, increasing verbal awareness was associated with fewer errors on the generation task. The awareness main effect was significant with both dependent variables for the letter stimuli and with the collapsed across trial blocks dependent variable for the asterisks stimuli, $\underline{F}(2,122)>7.12$. The aware groups made significantly fewer errors than the partial and unaware groups on both dependent variables with letters, $\underline{S}^{2}(2,122)>16.66$, and this difference approached significance with the asterisks data collapsed across trial blocks, $\underline{S}^{2}(2,128)=13.30, \underline{p}<0$ .002. Differences in errors made by the partial and unaware groups were not significant, $\underline{\mathrm{S}}^{2}(2,122)<2.70, \mathrm{p}>.05$.

Insert Table 7 about here 


\section{Awareness measures}

\section{Correlations between explicit measures}

If the measures derived from the three tasks used to assess awareness reflect a common explicit knowledge base acquired in training, then the measures should be correlated. The correlations between verbal awareness ratings; the difference between new and old, ringer and old, and ringer and new recognition part sequences; and generation errors on the first trial block were computed to assess the consistency of the explicit measures and are shown in Table 7. Explicit knowledge is reflected by: low verbal awareness scores; large difference scores between new-old recognition ratings and ringerold recognition ratings; minimal difference scores between ringer-new recognition ratings; and few generation errors on the first trial block. With the exception that participants who best discriminated ringer and old part sequences were more likely to erroneously discriminate ringer and new part sequences with both stimulus types, all the significant correlations are consistent with the assumption that there is a common explicit knowledge base reflected in each of the explicit measures. Inspection of the table reveals that the correlations between the recognition measures are particularly robust but the remaining correlations reflect considerable variability across measures. However, since verbal awareness is consistently related to all the measures there is no apparent reason not to use it as an individual difference index.

Insert Table 8 about here

\section{Perfect performance as a function of structure and awareness}

There are a number of criteria that could be used to categorize participants as explicit learners in a SRT task. A priori, perfect performance on either the generation or 
recognition task would seem to be particularly robust measures of explicit learning (e.g., Willingham et al., 1989, full explicit knowledge). The relationships between verbal awareness judgments, perfect performance on recognition tests, and perfect generation performance on the first block of trials are considered here. As can be seen in Table 8, if perfect performance on the 12 recognition test sequences was used as the criteria for explicit learning, 13 of 69 aware, 2 of 29 partially aware, and 2 of 30 unaware participants would have been judged as explicit learners with the letter sequences, while 14 of 62 aware, 2 of 23 partially aware, and 3 of 43 unaware participants would have been judged as explicit learners with the asterisk sequences. Only eight of these participants performed perfectly with both letter and asterisk sequences. If perfect recall of the sequence as reflected in motor responses on the first block of the generation task was used as the criteria for explicit learning, $13(9)$ aware, $1(0)$ partially aware, and $1(0)$ unaware would have been classified as explicit learners with the letter (asterisk) sequences. Only three of these participants performed perfectly with both letter and asterisk sequences. If perfect performance on both the recognition and generation task with letters (asterisks) was used as the criteria for explicit learning, six (four) participants would have been classified as explicit learners. Only one participant performed perfectly on all tasks. Obviously, the perfect performance measures are too restrictive to provide an adequate way to characterize participants as explicit learners. On the other hand, an inspection of the table highlights the interdependency of the verbal awareness ratings with both perfect performance measures. In addition, only verbally aware participants performed perfectly on both the recognition and generation tasks with either letters or asterisks. As is the case with the correlation data, these relationships reflect that performance on all three transfer measures is based, at least in part, on the explicit knowledge acquired during SRT learning. 
Discussion

Assessing Explicit Knowledge

\section{Verbal Reports as an Assessment of Implicit/Explicit Learning}

It has been argued that verbal reports are insensitive measures of explicit knowledge (Perruchet \& Amorin, 1992; Shanks \& St. John, 1994) that may not tap low confidence knowledge (Berry \& Dienes, 1993). Although this insensitivity may characterize the reports of some participants in SRT tasks, it appears that false positive reports were more likely than false negatives in the present experiment. Many children in the quasi-random group reported experiencing a structured sequence when such a sequence did not exist. Furthermore, the distribution of verbally aware, partially aware, and unaware responses were similar for children in the quasi-random and the moderate-structure groups. However, the verbal reports of children in both the moderate and high structure groups correlated with other performance measures such as SRT latency, recognition accuracy, and generation errors. It would appear that the children in the high and moderate structure groups based their verbal awareness reports on the accessibility of a representation of the repeating sequence. It is unlikely that children in these two groups would base their verbal reports on a representation of information acquired in the experimental session while children in the quasi-random group would just spuriously produce verbal awareness judgements. If this is the case, what information did the children in the quasi-random group use when they reported awareness or partial awareness?

A possible explanation for why some of the children in the quasi-random group reported awareness. The 10-trial repeating sequences were designed so that obvious 4-trial sequences did not appear. The quasi-random sequences were constructed so that stimuli did not repeat on successive trials so that if a particular stimulus appeared on Trial N, all other stimuli were equally likely to occur on Trial $\mathrm{N}+1$. These constraints generated 4-trial 
alternating sequences (i.e., XYXY, where $\mathrm{X}$ and $\mathrm{Y}$ represent any 2 stimuli) with a probability of $1 / 9$ and simple series (i.e., either $A B C D, B C D A, C D A B, D A B C, D C B A$, $\mathrm{CBAD}, \mathrm{BADC}$, or $\mathrm{ADCB}$ ) with a probability of $2 / 27$. Since these two types of simple patterns occurred on $18.5 \%$ of the 4-trial part sequences and were likely to be incidentally monitored and explicitly learned by the children in the quasi-random group, some of these children reported they could predict the stimuli in the SRT task.

\section{Recognition Memory Measures as Assessments of Implicit/Explicit Learning}

Meulemans et al. (1988) reported children and adults learned a repeating 10-trial sequence, but failed to recognize the difference between old and new 4-trial part sequences in a recognition task. Based on this finding, they claimed participants did not acquire any explicit knowledge in the SRT task. However, it was probably difficult to discriminate the new and old part-sequences because they only differed on the last trial. Thus, Meulemans et al. may have used an insensitive measure of participants' explicit knowledge. In order to increase the sensitivity of the recognition test, obvious ringer type sequences were added in the present experiment. If the children incidentally monitored the stimulus sequence for simple patterns such as perfectly ordered series (e.g., $\mathrm{ABCD}$ ), double alternations (e.g., $\mathrm{ABAB}$ ), double repetitions (e.g., $\mathrm{CCAA}$ ), and one stimulus appearing three times (e.g., $\mathrm{AABA}$ ), then the presence or the absence of such sequences should be recognized explicitly. Since the recognition task is considered an explicit task, children would be expected to access explicit representations more quickly and accurately than implicit representations. Consistent with these conjectures, children in the structured groups recognized the ringer, as compared to the old and new, sequences more quickly and accurately; and unaware and partially aware participants failed to discriminate the old and new sequences. However, the aware participants did discriminate the old and new sequences perhaps because the sequences used in the present experiment were less complex than those used by Meulemans et al. (1998). It would appear that if a range of sequences that are easy to discriminate are 
included in recognition tests, then the recognition task can be used to derive useful indices of implicit and explicit learning.

Intercorrelations between explicit measures

The correlations between the measures based on verbal awareness ratings, recognition judgements, and generation errors on the first trial block, for the most part, were consistent with the assumption that the explicit measures assess a common knowledge base acquired in SRT tasks. The frequency distributions based on awareness ratings, perfect performance on the recognition task, and perfect performance on the first block of the generation task reflect interdependencies that are also consistent with the idea of a common knowledge base. Although it has been argued that verbal reports are insensitive measures of explicit knowledge (Perruchet \& Amorin, 1992; Shanks \& St. John, 1994) that may not tap low confidence knowledge (Berry \& Dienes, 1993), results from the current experiment and those reported by Jackson and Jackson (1995) are inconsistent with these arguments as both children and adults who experienced quasi-random sequences reported they knew or sometimes knew where the next stimulus would appear. Thus, over-sensitivity, rather than undersensitivity might best characterize using verbal awareness ratings to index explicit knowledge. Nevertheless, the consistent association of verbal awareness ratings with all the measures derived from the recognition and generation tasks for children who experienced structured sequences support the continued use of these ratings as an index of individual differences.

\section{Age effects}

Reber $(1992,1993)$ argued that the cognitive hardware required for implicit processing predates the evolutionary development of the structures required for conscious awareness and, therefore, implicit learning should be age invariant, as compared to explicit learning which is age dependent (Siegler, 1998). Consistent with Reber's hypothesis: (a) 
the SRT curves of older and younger children were approximately parallel; (b) awareness ratings did not differ as a function of age; and (c) there was no significant difference in the number of errors on the recognition or generation task as a function of age. Based on these results and those reported by others (Meulemans et al. 1998; Thomas and Nelson, 2001), it appears that acquired implicit SRT knowledge is age invariant across middle childhood, 6 to 11 years. However, the failure of the children to learn the more complex structure in the present experiment and in the De Guise and Lassonde (2001) experiment, when contrasted with successful learning of comparable structures by older children (e.g., De Guise \& Lassonde) and adults (e.g., Stadler, 1992), relegates Reber's age invariance hypothesis to a relative short period of development.

\section{Sequence Structure Effects}

The verbal report and SRT latency data were consistent with the predictions that increasing structure would correlate with verbal awareness and learning rate. One possible explanation to account for this effect is that the children incidentally monitored and explicitly learned bigrams/dyads (e.g., AB, CA) in the repeating sequences. A repeating 5trial sequence was used in the high structure condition while a repeating 10 -trial sequence was used in the moderate structure condition. The defining 5-trials in the high structure sequence were identical to the first 5 trials of the moderate structure sequence. Therefore, not only did the children in the high structure, as compared to the moderate structure, group experience fewer unique dyads/bigrams, these dyads/bigrmas occurred at twice the frequency. If the children in the high structure group were more likely to detect and explicitly learn 2-trial sequences, then they should have quickly and accurately anticipated successive stimuli in both the SRT and generation task which, in turn, would have been reflected in decreasing SRT latencies across training and fewer generation errors. Consistent with these conjectures, participants in the high, as compared to the moderate, structure group learned at a faster rate in the SRT task and transferred that knowledge to the 
generation task. Surprisingly, structure did not affect performance of participants on the recognition task replicating the findings of Stadler and Neely (1997). It may be that only complete knowledge of the bigrams contained in each of the 4-trial part sequences is sufficient to facilitate recognition accuracy.

\section{Transfer}

Transfer of implicit knowledge across perceptual stimuli using the same response sequence

A number of investigators have found that implicitly acquired knowledge does not readily transfer across tasks or stimulus dimensions (Rabinowitz \& Herder, 2000; Rabinowitz \& Howe, 1994; Willingham et al., 1989). Such transfer specificity is considered to be a primary characteristic of implicit knowledge (Berry \& Dienes, 1993; Shanks \& Johnstone). However, Willingham (1999) found that when the same response location sequence was maintained, implicit sequence knowledge acquired by adults in a SRT task transferred across perceptual stimuli when the standard stimulus-response mapping was used, and also transferred across perceptual stimuli when the perceptual stimulus-response mapping was altered one space to the right of the location of the first response button. Willingham, concluded implicit sequence knowledge can be flexible.

Transfer of implicit knowledge was investigated in the present experiment by presenting participants a sequence of letters (asterisks) and then a sequence of asterisks (letters) while maintaining the same response location sequence. If acquired knowledge of the repeating sequence transferred across stimulus sequences, then SRTs on the second, as compared to the first, stimulus sequence should have been significantly faster. The only evidence that might be construed as knowledge transfer across perceptual stimuli appears in Table 3. Inspection of that table reveals that participants who experienced a particular perceptual stimulus set second responded faster with that set than did the participants who experienced the set first. This order effect was characteristic of children in the high and moderate structure groups with both letters and asterisks, and children in the quasi-random 
group with asterisks. Since only the children in the high structure condition improved in performance across trial blocks, the criterion Willingham et al. (1989) used as an index of SRT learning, and the order effect characterized the performance of the children in all groups with one exception, it most likely reflects nonspecific transfer rather than transfer of specific sequential information. No other significant contrast effects, across any dependent variables in both the general and awareness analyses, involved both stimulus-type sequence and stimulus type. Thus, it is unlikely that learning transferred from letters to asterisks or vice-versa.

Two explanations are offered as to why implicit transfer across stimulus sequences did not occur in the present experiment but did occur in two experiments reported by Willingham (1999). One possibility is that transfer of implicit knowledge may be age dependent. Consistent with this hypothesis, Reber (1967) found less implicit transfer with high school, as compared to university, students using an artifical grammar. Another, more speculative hypothesis, is that prior declarative knowledge may mediate transfer of implicitly acquired knowledge. Rabinowitz and Herder (2000) offered such a hypothesis to explain why verbally unaware children chose the nearest absolute stimulus on old conceptual dimensions, i.e., pictures of people representing an age dimension and sets of letters representing the alphabet, during transfer despite the fact that there was no clear physical relationship between these stimuli. It is possible Willingham (1999) obtained transfer from numbers to asterisks, in Experiment 2, because people have extensive experience counting stimuli that appear in a linear array and, therefore, automatically associated numbers with the positions at which the asterisks appeared. Similarly, extensive experience reading from left to right might have mediated the transfer when participants in Experiment 3 experienced a perceptual sequence of asterisks displaced one space to the left of the standard stimulus-response mapping and then experienced the same perceptual sequence properly aligned with the standard stimulus-response mapping. In contrast, 
children may not have had enough experience using letters to label stimuli in a linear array for declarative knowledge of associations between letters and positions to mediate transfer in a speeded implicit task.

Both implicit and explicit knowledge transfers across tasks

Although implicit knowledge did not transfer across stimulus dimensions in the present experiment it did transfer from the SRT task to the recognition task as children in the structured groups responded faster to the old part sequences than to the new and ringer part sequences. Explicit transfer occurred across tasks as structure was associated with transfer from the SRT to the generation task and awareness ratings were associated with both recognition judgments and generation errors. Thus, the implicit and explicit knowledge acquired in parallel during SRT learning (also see Curran and Keele, 1993; Noseworthy, 1996; Seger, 1994; Sloman, 1996; Willingham, 2001; Willingham \& GoedertEschmann, 1999; Willingham, Salidis, \& Gabrieli, 2002) were associated with different aspects of transfer performance.

\section{Summary}

1. Measures of explicit learning appear to be related to a common explicit knowledge base. Verbal awareness ratings were consistently associated with measures of explicit learning derived from the recognition and generation tasks and appear to be a useful index of explicit knowledge.

2. When children experience a quasi-random sequence, it appears that they incidently monitor and explicitly learn simple patterns. To the extent that their behaviour is characteristic of all participants, when children report they can predict the stimuli in the repeating sequence, their judgments are based on explicit knowledge of simple patterns rather than explicit knowledge of the entire sequence.

3. Children apparently incidently monitor and explicitly learn simple sequences while complex sequences are learned implicitly. Thus, both implicit and explicit learning 
occur in a SRT task, possibly in parallel.

4. Age only affected the speed of responding in the present experiment and other SRT studies in which comparably aged children were the participants. Thus, age invariance of implicit SRT learning characterizes middle childhood. Because older children and adults implicitly learn complex sequences in the SRT task while 6- to 11-year olds do not, Reber's $(1992,1993)$ age invariance of implicit learning hypothesis is inconsistent with SRT performance.

5. Children who experienced the same response location sequence but different perceptual sequences did not transfer acquired sequence knowledge across the different stimulus type sequences. It appears implicit learning by young school age children can be transfer specific when the same response sequence is experienced. 


\section{References}

Altmann, G. T. M., Dienes, Z., \& Goode., A. (1995). A modality independence of implicitly learned grammatical knowledge. Journal of Experimental Psychology: Learning. Memory, \& Cognition, 21, 4, 899-912.

Berry, D. C., \& Dienes, Z. (1993). Implicit Learning: Theoretical and Empirical Issues, Hove, U.K.:Erlbaum.

Cherry, K. E., \& Stadler, M. A. (1995). Implicit learning of a nonverbal sequence in younger and older adults. Psychology and Aging, 10, 3, 379-394.

Cohen, A., Ivry, R. I., \& Keele, S. W. (1990). Attention and structure in sequence learning. Journal of Experimental Psychology: Learning, Memory, and Cognition, 16, 1, 17 30.

Cohen, N. J., \& Poldrack, R. A. (1997). Memory for items and memory for relations in the procedural/declarative memory framework. Memory, 5, 1/2, 131-178.

Curran, T. (1997). Effects of aging on implicit sequence learning: Accounting for sequence structure and explicit knowledge. Psychological Research, 60, 24-41.

Curran, T., \& Keele, S. W. (1993). Attentional and nonattentional forms of sequence learning. Journal of Experimental Psychology: Learning. Memory, and Cognition. 19, 1 , 189-202.

De Guise, E., Jambaque, I., Dulac, O., Lassonde, M. (1999). Absence of procedural learning in children with frontal damage. Brain and Cognition, 40, 1, 90 - 94.

De Guise, E., Maryse, L. (2001). Callosal contribution to procedural learning in children. Developmental Neuropsychology, 19, 13, 253 - 272.

Fletcher, J., Maybery, M. T., Bennett, S. (2000). Implicit learning differences: A question of developmental level? Journal of Experimental Psychology: Learning, Memory and Cognition, 26, 1, $246-252$. 
Frensch, P., A., \& Miner, C., S. (1994). Effects of presentation rate and individual differences in short-term memory capacity on an indirect measure of serial learning. Memory \& Cognition, 22, 1, 95-110.

Hartman, M., Knopman, D. S., \& Nissen, M. J. (1989). Implicit learning of new verbal associations. Journal of Experimental Psychology: Learning, Memory, and Cognition, 15, 6, 1070-1082.

Howard, D. V., \& Howard, J. H. (1989). Age differences in learning serial patterns: Direct versus indirect measures. Psychology and Aging, 4, 3, 357-364.

Howard, D. V., \& Howard, J. H. (1992). Adult age differences in the rate of learning serial patterns: Evidence from direct and indirect tests. Psychology and Aging. 7, 2, 232241.

Howard, D. V., \& Howard, J. H. (2001). When it does hurt to try: Adult age differences in the effects of instructions on implicit pattern learning. Psychonomic Bulletin \& Review, 8, 4, 798-805.

Howard, J. H, \& Howard, D. V. (1997). Age differences in implicit learning of higher order dependencies in serial patterns. Psychology \& Aging, 12, 4, 634-656.

Howard, J. H., Mutter, S. A., Howard, D. V. (1992). Serial pattern learning by event observation. Journal of Experimental Psychology: Learning, memory, and Cognition, 18, 5, 1029-1039.

Inui, N. \& Suzuki, K. (1998). Practice and serial reaction time of adolescents with autism. Perceptual and Motor Skills, 86, 403-410.

Jackson, G. M. \& Jackson, S. R.(1995). Do measures of explicit learning actually measure what is being learnt? A critique of current methods. Psyche: An Interdisciplinary Journal of Research on Consciousness, 2, 20, NP. 
Japikse, K. C., Negash, S., Howard, J. H., Howard, D. V. (2003). Intermanual transfer of procedural learning after extended practice of probabilistic sequences. Experimental Brain Research, 148, 38-49.

Johnson, M.H. (1997). Developmental Cognitive Neuroscience. An introduction. Cambridge, MA: Blackwell.

Keel, S.W., Jennings, P., Jones, S., Caulton, D., \& Cohen, A. (1995). On the modularity of sequence representation. Journal of Motor Behavior, 27, 17-30.

Kelly, S. W. \& Burton, A. M. (2001). Learning complex sequences: no role for observation? Psychological Research, 65, 15-23.

Knopman, D. \& Nissen, M.. J. (1991). Procedural learning is impaired in Huntington's disease: Evidence from the serial reaction time task. Neuropsychologia, 22, 3, 245-254.

Laforce, R., Hayward, S., \& Cox, L. V. (2001). Impaired skill learning in children with heavy prenatal alcohol exposure. Journal of the International Neuropsychological Society, 7, 112-114.

Litke, D. R. (2001). Implicit learning and development. (Doctoral dissertation, Long Island University, 2001). Dissertation Abstracts International, 61, 11, 6159.

Loftus, E, F., \& Klinger, M. R. (1992). Is the unconscious smart or dumb? American Psychologist. 47, 6, 761-765.

Mathews, R. C., Buss, R. R., Stanley, W. B., Blanchard-Fields, F., Cho, J. R., \& Druhan, B. (1989). Role of implicit and explicit processes in learning from examples: A synergistic effect. Journal of Experimental Psychology: Learning, Memory, \& Cognition, $15,6,1083-1100$.

Maybery, M., Taylor, M., \& O'Brien-Malone, A. (1995). Implicit learning: Sensitive to age but not IQ. Australian Journal of Psychology. 47, 1, 8-17. 
Mayr, U. (1996). Spatial attention and implicit sequence learning evidence for independent learning of spatial and nonspatial sequences. Journal of Experimental Psychology: Learning, Memory, and Cognition, 22, 2, 350-364.

Meulemans, T., \& Van der Linden, M. (1998). Implicit sequence learning in children. Journal of Experimental Child Psychology, 69, 199-221.

Nissen, M. J., \& Bullemer, P. (1987). Attentional requirements of learning: Evidence from performance measures. Cognitive Psychology, 19, 1-13.

Nissen, M., J., Willingham, D. B., \& Hartman, M. (1989). Explicit and implicit remembering: When is learning preserved in amnesia? Neuropsychologia, 27, 3, 341-352.

Noseworthy, J. (1994). Acquisition of covariation information and verbal awareness in elementary school children. Masters Thesis, Memorial University of Newfoundland.

Perruchet, P., \& Amorim, M. A. (1992). Conscious knowledge and changes in performance in sequence learning: Evidence Against dissociation. Journal of Experimental Psychology: Learning, Memory and Cognition, 18, 4, 785-800.

Perruchet, P., \& Pacteau, C. (1990). Synthetic grammar learning: Implicit rule abstraction or explicit fragmentary knowledge. Journal of Experimental Psychology: General, 119, 3, 264-275.

Rabinowitz, F. M., \& Herder, M. (2000). Implicit and explicit learning of the middle concept: Different transfer functions. Masters Thesis, Memorial University of Newfoundland.

Rabinowitz, F. M., \& Howe, M. L. (1994). Development of the middle concept. Journal of Experimental Child Psychology, 57, 418-448.

Reber, A. S. (1967). Implicit learning of artificial grammar. Journal of Verbal Awareness and Verbal Behavior, 6, 855-863.

Reber, A.S. (1969). Transfer of syntactic structure in synthetic languages. Experimental Psychology. 81, 1, 115-119. 
Reber, A. S. (1992). The cognitive unconscious: An evolutionary perspective. Consciousness and Cognition, 1, 93-133.

Reber, A.S. (1993). Implicit learning and tacit knowledge: An essay on the cognitive unconscious. NY: Clarendon Press.

Reingold, E. M., \& Merikle, P. M. (1988). Using direct and indirect measures to study perception without awareness. Perception and Psychophysics, 44, 563-575.

Rovee-Collier, C., Hayne, H., Columbo, M. (2001). The development of implicit and explicit memory. Amsterdam: John Benjamins

Scheffé, H. (1959). The analysis of variance. New York:Wiley.

Seger, C. A., (1994). Implicit learning._Psychological Bulleting 115, 2, 163-196.

Shanks, D. R., \& St. John, M. F. (1994). Characteristics of dissociable human learning systems. Behavioral and Brain Sciences, 17, 367-447.

Shanks, D. R., \& Johnstone, T. (1999). Evaluating the relationship between explicit and implicit knowledge in a sequential reaction time task. Journal of Experimental Psychology: Learning, Memory, and Cognition. 25, 6, 1435-1451.

Siegler, R. S. (1998). Children's thinking ( $3^{\text {rd }}$ ed.). New Jersey:Prentice Hall.

Sloman, S. A., (1996). The empirical case for two systems of reasoning. Psychological Bulletin, 119, 1, 3-22.

Stadler, M. A.(1992). Statistical structure and implicit serial learning. Journal of Experimental Psychology: Learning, Memory, \& Cognition, 18, 2, 318-327.

Stadler, M.A., \& Neely, C. B. (1997). Effects of sequence length and structure on implicit serial learning. Psychological Research, 60, 14-23.

Stadler, M. A., \& Roediger III, H.L. (1998). The question of awareness in research on implicit learning. In M.A. Stadler \& P. A. Frensch (Eds.), Handbook of Implicit Leaning (pp. 105 132). Thousand Oaks, California: Sage. 
Tunney, R. J., \& Altmann, G. T. M. (1999). The transfer effect in artificial grammar learning: Reappraising the evidence on the transfer of sequential dependencies. Journal of Experimental Psychology: Learning, Memory, \& Cognition, 25, 5,1322-1333.

Thomas, K., M., \& Nelson, C., A. (2001). Serial reaction time learning in preschooland school-age children. Journal of Experimental Child Psychology, 79, 4, 364-387.

Vinter, A. \& Perruchet, P. (2000). Implicit learning in children is not related to age: Evidence from drawing behavior. Child Development, 71, 5, 1223-1240.

Vinter, A. \& Perruchet, P. (2002). Implicit motor learning through observational training in adults and children. Memory and Cognition, 30, 2, 256-261.

Willingham, D. B. (1997). Implicit and explicit memory do not differ in flexibility: Comment on Dienes and Berry (1997). Psychonomic Bulletin and Review 4, 4, 587-591.

Willingham, D. B., (1998a). A neuropsychological theory of motor skill learning. Psychological Review, 105, 3, 558-584.

Willingham, D. B. (1998b). What differentiates declarative and procedural memories: Reply to Cohen, Poldrack, and Eichenbaum (1997). Memory, 6, 6, 689 - 699.

Willingham, D. B., (1999). Implicit motor sequence learning is not purely perceptual. Memory and Cognition, 27, 3, 561 - 572.

Willingham, D. B. (2001). Becoming aware of motor skill. Trends in Cognitive Science, $5,5,181-182$.

Willingham, D. B., \& Dumas, J. A. (1997). Long-term retention of a motor skill: Implicit sequence knowledge is not retained after a one-year delay. Psychological Research. 60, $113-119$.

Willingham, D. B., Greeley, T., \& Bardone, A. M. (1993). Dissociation in a serial response time task using a recognition measure: Comment on Perruchet and Amorim. Journal of Experimental Psychology: Learning, Memory, and Cognition, 19, 6, 1424-1430. 
Willingham, D. B., Nissen, M. J., \& Bullemer, P. (1989). On the development of procedural knowledge. Journal of Experimental Psychology: Learning, Memory, and Cognition, 15, 6, 1047-1060.

Willingham, D. B., Salidis, J., Gabrieli, J. D. E. (2002). Direct comparison of neural systems mediating conscious and unconscious skill learning. Journal of Neurophysiology, $\underline{88}, 1451-1460$.

Willingham, D. B., Wells, L. A., Farrell, J. M., \& Stemwedel, M. E. (2000). Implicit motor sequence learning is represented in response locations. Memory and Cognition, 28, 3, 366-375.

Zumbo, B. D., \& Zimmerman, D. W. (1993). Is the selection of statistical methods governed by level of measurement? Canadian Psychology, 34, 4, 390-400. 
Table 1

Summary of experimental design.

\begin{tabular}{|c|c|c|c|c|c|c|c|}
\hline 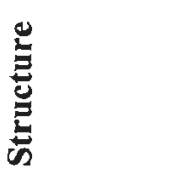 & 苋 & 总 & 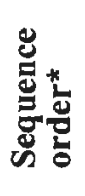 & SRT Task & $\begin{array}{l}\text { Verbal } \\
\text { Awareness } \\
\text { Task }\end{array}$ & $\begin{array}{l}\text { Recognition } \\
\text { Task }\end{array}$ & $\begin{array}{l}\text { Generation } \\
\text { Task }\end{array}$ \\
\hline $\begin{array}{l}\text { Quasi } \\
\text { Random }\end{array}$ & 3,6 & $M, F$ & 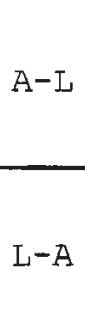 & $\begin{array}{l}\text { Children } \\
\text { responded } \\
\text { to a quasi } \\
\text { random } \\
\text { sequence. }\end{array}$ & $\begin{array}{l}\text { Children } \\
\text { questioned } \\
\text { on ability } \\
\text { to predict } \\
\text { stimuli. }\end{array}$ & & \\
\hline Moderate & 3,6 & $M, F$ & 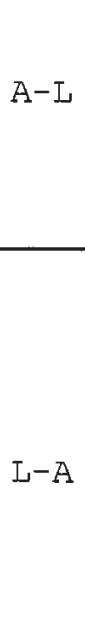 & $\begin{array}{l}\text { Children } \\
\text { responded } \\
\text { to a } \\
\text { moderate } \\
\text { structure } \\
\text { sequence. }\end{array}$ & $\begin{array}{l}\text { Children } \\
\text { questioned } \\
\text { on ability } \\
\text { to predict } \\
\text { stimuli. }\end{array}$ & $\begin{array}{l}\text { Children } \\
\text { asked to } \\
\text { rate their } \\
\text { recognition } \\
\text { of old, } \\
\text { new, and } \\
\text { ringer 4- } \\
\text { trial part } \\
\text { sequences }\end{array}$ & $\begin{array}{l}\text { Children } \\
\text { were } \\
\text { presented } \\
\text { the first } \\
\text { stimulus in } \\
\text { the sequence } \\
\text { and asked to } \\
\text { predict the } \\
\text { next } 39 \text {. }\end{array}$ \\
\hline $\mathrm{High}$ & 3,6 & $M, E$ & 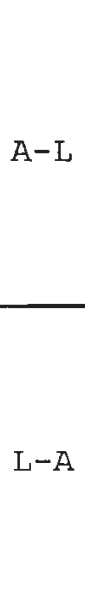 & $\begin{array}{l}\text { Children } \\
\text { responded } \\
\text { to a high } \\
\text { structure } \\
\text { sequence. }\end{array}$ & $\begin{array}{l}\text { Children } \\
\text { questioned } \\
\text { on ability } \\
\text { to predict } \\
\text { stimuli. }\end{array}$ & $\begin{array}{l}\text { Children } \\
\text { asked to } \\
\text { rate their } \\
\text { recognition } \\
\text { of old, } \\
\text { new, and } \\
\text { ringer 4- } \\
\text { trial part } \\
\text { sequences }\end{array}$ & $\begin{array}{l}\text { Children } \\
\text { were } \\
\text { presented } \\
\text { the first } \\
\text { stimulus in } \\
\text { the sequence } \\
\text { and asked to } \\
\text { predict the } \\
\text { next } 39 .\end{array}$ \\
\hline
\end{tabular}

A-L means participants experienced asterisks first then letters

L-A means participants experienced letters first then asterisks 
Table 2

Number of children reporting they were aware, partially aware, or unaware of the sequential structure.

Structure

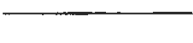

Quasi-random

Moderate

High
Letters

\begin{tabular}{ccc}
\hline Aware & Partial & Unaware \\
10 & 16 & 22 \\
19 & 21 & 24 \\
50 & 8 & 6
\end{tabular}

Asterisks

$\begin{array}{ccc}\text { Aware } & \text { Partial } & \text { Unaware } \\ 6 & 12 & 30 \\ 19 & 15 & 30 \\ 43 & 8 & 13\end{array}$


Table 3

Mean (SD) serial reaction time in seconds for each seguential structure group as a function of stimulus-type sequence and the order in which each stimulus sequence was experienced.

\begin{tabular}{|c|c|c|c|c|}
\hline Stimulus Type & Structure & & Order & \\
\hline & & First & Second & Means (SD) \\
\hline Letters & Quasi-random & $0.90(0.20)$ & $0.95(0.20)$ & $0.93(0.20)$ \\
\hline & Moderate & $0.85(0.19)$ & $0.79(0.23)$ & $0.82(0.21)$ \\
\hline & High & $0.74(0.29)$ & $0.59(0.32)$ & $0.66(0.31)$ \\
\hline & Means (SD) & $0.82(0.24)$ & $0.76(0.30)$ & $0.79(0.27)$ \\
\hline & & Eirst & Second & Means (SD) \\
\hline Asterisks & Quasi-random & $0.80(0.17)$ & $0.74(0.19)$ & $0.77(0.18)$ \\
\hline & Moderate & $0.72(0.24)$ & $0.67(0.22)$ & $0.70(0.23)$ \\
\hline & $\mathrm{High}$ & $0.65(0.22)$ & $0.55(0.25)$ & $0.60(0.24)$ \\
\hline & Means (SD) & $0.72(0.22)$ & $0.65(0.24)$ & $0.68(0.23)$ \\
\hline
\end{tabular}


Table 4

Number of SRT errors across trial blocks as a function of stimulus type.

Stimulus type

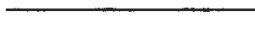

Asterisks

Letters

Means
Trial Block

1

4
2.53

3.08

3

3.52

2.68

2.80

3.06

3.10

2.94

Means

3.11

2.77

2.62

2.99

3.06 


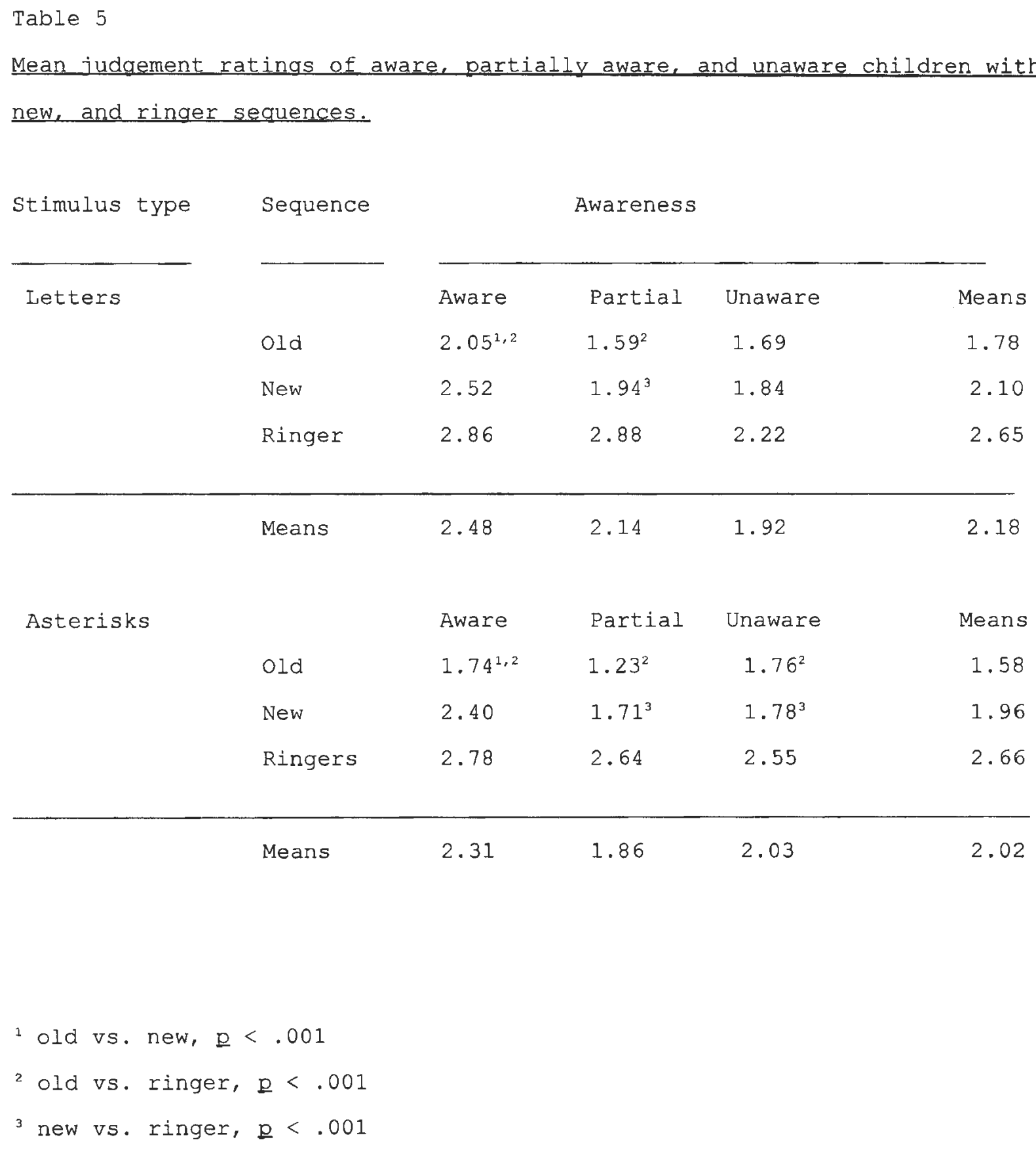


Table 6

Number of generation errors of aware, partially aware, and unaware children averaged across each 10-trial block.

\begin{tabular}{|c|c|c|c|c|c|c|}
\hline \multirow{2}{*}{ Stimulus type } & \multirow[t]{2}{*}{ Awareness } & \multicolumn{5}{|c|}{ Trial Blocks } \\
\hline & & 1 & 2 & 3 & 4 & Means \\
\hline & Aware & 4.16 & 3.89 & 3.02 & 2.70 & 3,44 \\
\hline & Partial & 6.26 & 6.83 & 6.09 & 5.69 & 6.22 \\
\hline & Unaware & 8.35 & 7.81 & 8.54 & 7.13 & 7.96 \\
\hline & Means & 6.26 & 6.18 & 5.88 & 5.17 & 5.87 \\
\hline
\end{tabular}

Asterisks

1

Means

Aware

6.21

4.11

4. 32

4.00

4.66

Partial

7.69

6.55

5.06

5.48

6.20

Unaware

8.63

6.95

7.58

7.46

7.66

Means

7.51

5.87

5.66

5.65

6.17 
Table 7

The correlation between explicit measures.

\begin{tabular}{lccccc} 
& & \multicolumn{2}{c}{$\frac{\text { Letters }}{}$} & \\
& verb awa & new-old & ring-old & ring-new & gen err TB1 \\
verb awa & 1.00 & & & & \\
new-old & -0.11 & 1.00 & & & \\
ring-old & 0.06 & $0.60 * *$ & 1.00 & 1.00 & \\
ring-new & $0.18^{*}$ & $-0.43^{*}$ & $0.47 * *$ & $0.18 *$ & 1.00 \\
gen err TB1 & $0.45^{*}$ & $-0.25^{*}$ & -0.09 & 0.00
\end{tabular}

Asterisks

verb awa $\quad 1.00$

new-old $\quad-0.31^{\star *} \quad 1.00$

ring-old $\quad-0.07 \quad 0.53^{\star \star} \quad 1.00$

$\begin{array}{lllll}\text { ring-new } & 0.25^{*} & -0.48^{* *} & 0.49 * * & 1.00\end{array}$

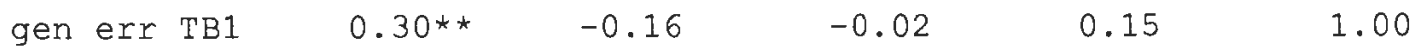

Note ${ }^{\star} p<.05,{ }^{\star \star} \mathrm{p}<.01$ 
Table 8

The frequency of participants who were errorless on the recognition task or on the first trial block of the generation task.

Task

Recognition
Stimulus Type structure

Letters

(n)

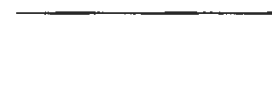

Moderate

High Asterisks

Moderate
High

High

Letters

Generation

$\begin{array}{ll}\text { Moderate } & \text { High } \\ \text { Asterisks } & \text { Moderate } \\ \text { High }\end{array}$

\begin{abstract}
Aware
\end{abstract}
1

$12^{a}$

Aware Partial

1

$8^{b}$

5

$9^{\text {b }}$

Awareness

\begin{tabular}{ccc}
\hline Aware & Partial & Unaware \\
2 & 2 & 2 \\
$11^{a}$ & 0 & 0 \\
\end{tabular}

Aware

$\begin{array}{cc}\text { Partial } & \text { Unaw } \\ 2 & \\ 0 & 0\end{array}$

3

0

ware

(1)




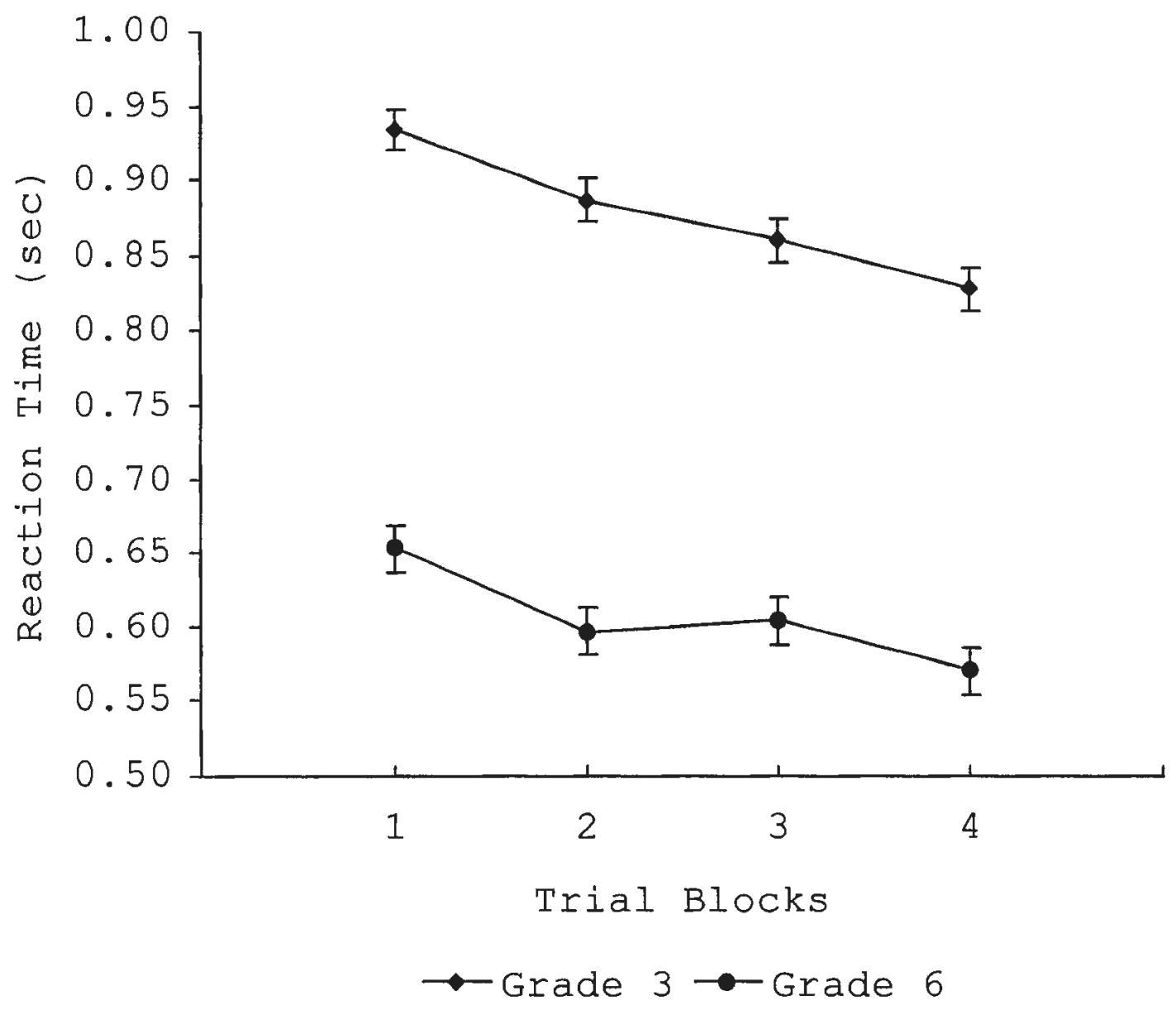

Figure 1. The SRT task average median RT on each trial block as a function of age. 


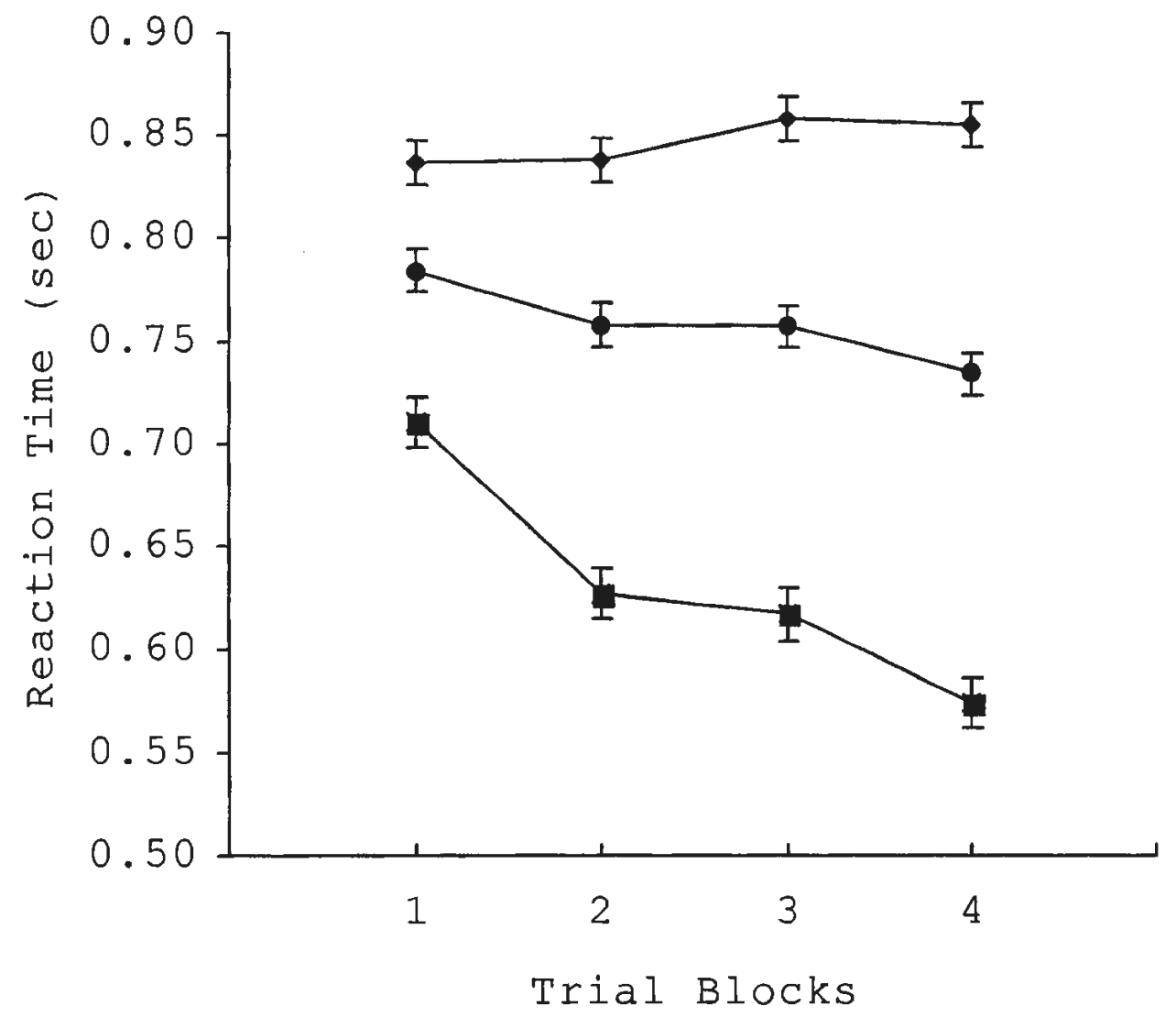

$\rightarrow$ Quasi random $\rightarrow$ Moderate $\rightarrow-$ High

Eigure 2. The SRT task average median RT on each trial block as a function of sequence structure. 


